\title{
Two species competitive model with the Allee effect
}

\section{Ann Brett and Mustafa RS Kulenović}

"Correspondence:

kulenm@math.uri.edu

Department of Mathematics,

University of Rhode Island, Kingston,

RI 02881-0816, USA

\begin{abstract}
We consider the following system of difference equations: $x_{n+1}=\frac{a x_{n}^{2}}{1+x_{n}^{2}+c y_{n}}$, $y_{n+1}=\frac{b y_{n}^{2}}{1+y_{n}^{2}+d x_{n}}, n=0,1, \ldots$, where $a, b, c, d$ are positive constants and $x_{0}, y_{0} \geq 0$ are initial conditions. This system has interesting dynamics and can have up to nine equilibrium points. The most complex and perhaps most interesting case is the case of nine equilibrium points, four of which are local attractors, four of which are saddle points, and one of which is a repeller. Using recent results of Kulenović and Merino we are able to characterize the basins of attractions of all local attractors and thus to describe the global dynamics of this system. This case can be considered as a two-dimensional version of the Allee effect for competitive systems.
\end{abstract}

MSC: 39A10; 39A30; 37G35

Keywords: Allee effect; basin; competition; difference equation; global asymptotic stability; invariant manifold; stable manifold

\section{Introduction}

The following difference equation is known as the Beverton-Holt model:

$$
x_{n+1}=\frac{a x_{n}}{1+x_{n}}, \quad n=0,1, \ldots
$$

where $a>0$ is the rate of change (growth or decay) and $x_{n}$ is the size of the population at the $n$th generation.

This model was introduced by Beverton and Holt in 1957. It depicts density dependent recruitment of a population with limited resources which are not shared equally. The model assumes that the per capita number of offspring is inversely proportional to a linearly increasing function of the number of adults. In other words (1) can be considered as an equation of the form

$$
x_{n+1}=x_{n} f\left(x_{n}\right), \quad n=0,1, \ldots,
$$

where $f(u)=a /(1+u)$ is inversely proportional to the linear function $A+B u, A, B>0$, which can be normalized to be $1+u$.

The Beverton-Holt model is well studied and understood. It exhibits the following properties.

O2014 Brett and Kulenović; licensee Springer. This is an Open Access article distributed under the terms of the Creative Commons Attribution License (http://creativecommons.org/licenses/by/2.0), which permits unrestricted use, distribution, and reproduction in any medium, provided the original work is properly cited. 
Theorem 1 Equation (1) has the two equilibrium points 0 and $a-1$ when $a>1$.

(a) All solutions of (1) are monotonic (increasing or decreasing) sequences.

(b) If $a \leq 1$, then the zero equilibrium is a global attractor, that is, $\lim _{n \rightarrow \infty} x_{n}=0$, for all $x_{0} \geq 0$.

(c) If $a>1$, then the equilibrium point $a-1$ is a global attractor, that is, $\lim _{n \rightarrow \infty} x_{n}=a-1$, for all $x_{0}>0$.

(d) Both equilibrium points are globally asymptotically stable in the corresponding regions of parameters $a \leq 1$ and $a>1$, that is, they are global attractors with the property that small changes of initial condition $x_{0}$ result in small changes of the corresponding solution $\left\{x_{n}\right\}$.

Furthermore, (1) can be solved explicitly and has the following solution:

$$
\begin{aligned}
& x_{n}=\frac{1}{1 /(a-1)+\left(1 / x_{0}-1 /(a-1)\right) 1 / a^{n}} \quad \text { if } a \neq 1, \\
& x_{n}=\frac{1}{n+1 / x_{0}} \quad \text { if } a=1,
\end{aligned}
$$

which can be used to prove all the preceding properties. See [1-4].

The Allee effect is a phenomenon in biology characterized by a positive correlation between population density and per capita growth rate. The Allee effect was first written on extensively by its namesake Warder Clyde Allee. The general idea of the Allee effect is that for smaller populations, the reproduction and survival rates of individuals decrease. This effect usually saturates or disappears as populations get larger. The Allee effect has been detected in a number of discrete models; see [4-7].

The effect may be due to any number of causes. In some species, reproduction (finding a mate in particular) may be increasingly difficult as the population density decreases.

In mathematics, when the basin of attraction of the zero equilibrium of a system contains an open set, we consider the system to exhibit the Allee effect. See $[4,5]$.

In view of Theorem 1 the Beverton-Holt model does not exhibit the Allee effect.

\subsection{Beverton-Holt type model that exhibits the Allee effect}

The difference equation

$$
x_{n+1}=\frac{a x_{n}^{2}}{1+x_{n}^{2}}, \quad n=0,1, \ldots
$$

which was introduced by Thompson [8] as a depensatory generalization of the BevertonHolt stock-recruitment relationship, was used to develop a set of constraints designed to safeguard against overfishing. This model has been used in the study of fish population dynamics, particularly when overfishing is present; see [7] for further references. In view of the sigmoid shape of the function $f(u)=\frac{a u^{2}}{1+u^{2}}$, (4) is called the sigmoid Beverton-Holt model. A very important feature of the sigmoid Beverton-Holt model is that it exhibits the Allee effect. We can see this from the following result, the proof of which is an immediate consequence of a stair-step diagram analysis.

Theorem 2 The global dynamics of Equation (4) is as follows: 
(a) Equation (4) has only a zero equilibrium when $a<2$.

(b) Equation (4) has a zero equilibrium and the positive equilibrium $\bar{x}=1 / 2$, when $a=2$.

(c) There exists a zero equilibrium and two positive equilibria, $\bar{x}_{-}$and $\bar{x}_{+}$, when $a>2$.

(d) All solutions of (4) are monotonic (increasing or decreasing) sequences.

(e) If $a<2$, then the equilibrium point 0 is a global attractor, that is, $\lim _{n \rightarrow \infty} x_{n}=0$ for all $x_{0} \geq 0$.

(f) If $a=2$, then the equilibrium point 0 is a global attractor, with the basin of attraction $B(0)=(0, \bar{x})$ and $\bar{x}=1 / 2$ is a non-hyperbolic equilibrium point with the basin of attraction $B(\bar{x})=[\bar{x}, \infty)$.

(g) If $a>2$, then we have zero equilibrium and $\bar{x}_{+}$are locally asymptotically stable, while $\bar{x}_{-}$is a repeller and the basins of attraction of the equilibrium points are given as

$$
\begin{aligned}
& B(0)=\left\{x_{0}: 0 \leq x_{0}<\bar{x}_{-}\right\}, \\
& B\left(\bar{x}_{+}\right)=\left\{x_{0}: \bar{x}_{-}<x_{0}<\infty\right\} .
\end{aligned}
$$

In other words, the smaller positive equilibrium serves as the boundary between two basins of attraction. The zero equilibrium has the basin of attraction $B(0)$ and the model exhibits the Allee effect.

(h) The equilibrium points 0 and $\bar{x}_{+}$are globally asymptotically stable in the corresponding basins of attractions $B(0)$ and $B\left(\bar{x}_{+}\right)$.

\subsection{Competitive model in two dimensions that exhibits the Allee effect}

We will now consider the two-dimensional analog of (4) which is the uncoupled system

$$
\begin{aligned}
& x_{n+1}=\frac{a x_{n}^{2}}{1+x_{n}^{2}}, \\
& y_{n+1}=\frac{b y_{n}^{2}}{1+y_{n}^{2}}, \quad n=0,1, \ldots,
\end{aligned}
$$

where $a$ and $b$ are positive parameters. System (5) can have at most nine nonnegative equilibrium points:

$$
\begin{array}{lrc}
E_{0}(0,0), & E_{\bar{y}_{-}}\left(0, \bar{y}_{-}\right), & E_{\bar{y}_{+}}\left(0, \bar{y}_{+}\right), \\
E_{\bar{x}_{-}}\left(\bar{x}_{-}, 0\right), & E_{\bar{x}_{+}}\left(\bar{x}_{+}, 0\right), & E_{-}\left(\bar{x}_{-}, \bar{y}_{-}\right), \\
E_{-+}\left(\bar{x}_{-}, \bar{y}_{+}\right), & E_{+-}\left(\bar{x}_{+}, \bar{y}_{-}\right), & E_{+}\left(\bar{x}_{+}, \bar{y}_{+}\right) .
\end{array}
$$

The dynamics of system (5) is partially described in the following theorem, with complete visual interpretation in Figure 1 can be derived from Theorem 2. Since there are three dynamics scenarios for each of two equations in (5), there are nine dynamic scenarios for system (5), which are visualized in Figure 1.

Theorem 3 System (5) has the following properties:

(a) All solutions $\left(x_{n}, y_{n}\right)$ of system (5) are component-wise monotonic and bounded, that is, both sequences $\left\{x_{n}\right\}$ and $\left\{y_{n}\right\}$ are monotonic and bounded.

(b) If $a<2$ and $b<2$, then there exists only a zero equilibrium, which is a global attractor. 

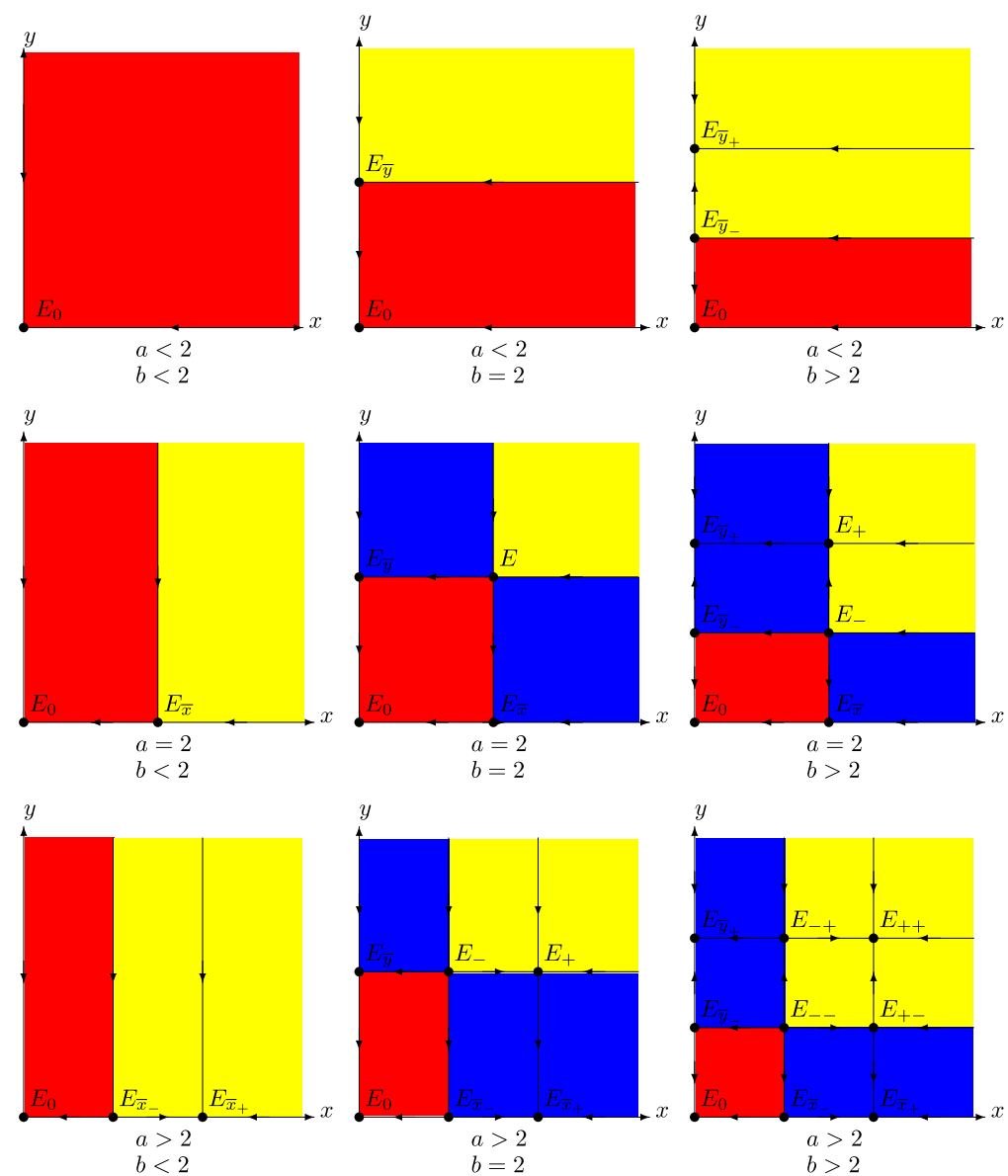

Figure 1 Global dynamics of uncoupled two-dimensional model (5) with nine dynamics scenarios.

(c) If $a<2, b>2$, then the basins of attraction of the equilibrium points are given as

$$
\begin{aligned}
& B\left(E_{0}(0,0)\right)=\left\{\left(x_{0}, y_{0}\right): 0 \leq x_{0}<\infty, 0 \leq y_{0}<\bar{y}_{-}\right\}, \\
& B\left(E_{\bar{y}_{+}}\left(0, \bar{y}_{+}\right)\right)=\left\{\left(x_{0}, y_{0}\right): 0 \leq x_{0}<\infty, \bar{y}_{-}<y_{0}<\infty\right\} .
\end{aligned}
$$

(d) If $a>2, b<2$, then the basins of attraction of the equilibrium points are given as

$$
\begin{aligned}
& B\left(E_{0}(0,0)\right)=\left\{\left(x_{0}, y_{0}\right): 0 \leq x_{0}<\bar{x}_{-}, 0 \leq y_{0}<\infty\right\}, \\
& B\left(E_{\bar{x}_{+}}\left(\bar{x}_{+}, 0\right)\right)=\left\{\left(x_{0}, y_{0}\right): \bar{x}_{-}<x_{0}<\infty, 0 \leq y_{0}<\infty\right\} .
\end{aligned}
$$

(e) If $a>2, b>2$, then the basins of attraction of the equilibrium points are given as

$$
\begin{aligned}
& B\left(E_{0}(0,0)\right)=\left\{\left(x_{0}, y_{0}\right): 0 \leq x_{0}<\bar{x}_{-}, 0 \leq y_{0}<\bar{y}_{-}\right\}, \\
& B\left(E_{\bar{x}_{+}}\left(\bar{x}_{+}, 0\right)\right)=\left\{\left(x_{0}, y_{0}\right): \bar{x}_{-}<x_{0}<\infty, 0 \leq y_{0}<\bar{y}_{-}\right\}, \\
& B\left(E_{\bar{y}_{+}}\left(0, \bar{y}_{+}\right)\right)=\left\{\left(x_{0}, y_{0}\right): 0 \leq x_{0}<\bar{x}_{-}, \bar{y}_{-}<y_{0}<\infty\right\}, \\
& B\left(E_{++}\left(\bar{x}_{+}, \bar{y}_{+}\right)\right)=\left\{\left(x_{0}, y_{0}\right): \bar{x}_{-}<x_{0}<\infty, \bar{y}_{-}<y_{0}<\infty\right\}, \\
& B\left(E_{\bar{x}_{-}}\left(\bar{x}_{-}, 0\right)\right)=\left\{\left(\bar{x}_{-}, y_{0}\right): 0 \leq y_{0}<\bar{y}_{-}\right\},
\end{aligned}
$$




$$
\begin{aligned}
& B\left(E_{\bar{y}_{-}}\left(0, \bar{y}_{-}\right)\right)=\left\{\left(x_{0}, \bar{y}_{-}\right): 0 \leq x_{0}<\bar{x}_{-}\right\}, \\
& B\left(E_{+-}\right)=\left\{\left(x_{0}, \bar{y}_{-}\right): \bar{x}_{-}<x_{0}<\infty\right\}, \quad B\left(E_{-+}\right)=\left\{\left(\bar{x}_{-}, y_{0}\right): \bar{y}_{-}<y_{0}<\infty\right\} .
\end{aligned}
$$

(f) If $a=2, b<2$, then the basins of attraction of the equilibrium points are given as

$$
\begin{aligned}
& B\left(E_{0}(0,0)\right)=\left\{\left(x_{0}, y_{0}\right): 0 \leq x_{0}<\bar{x}, 0 \leq y_{0}<\infty\right\}, \\
& B\left(E_{\bar{x}}(\bar{x}, 0)\right)=\left\{\left(x_{0}, y_{0}\right): \bar{x} \leq x_{0}<\infty, 0 \leq y_{0}<\infty\right\} .
\end{aligned}
$$

(g) If $a<2, b=2$, then the basins of attraction of the equilibrium points are given as

$$
\begin{aligned}
& B\left(E_{0}(0,0)\right)=\left\{\left(x_{0}, y_{0}\right): 0 \leq x_{0}<\infty, 0 \leq y_{0}<\bar{y}\right\}, \\
& B\left(E_{\bar{y}}(0, \bar{y})\right)=\left\{\left(x_{0}, y_{0}\right): 0 \leq x_{0}<\infty, \bar{y} \leq y_{0}<\infty\right\} .
\end{aligned}
$$

(g) If $a=2, b=2$, then the basins of attraction of the equilibrium points are given as

$$
\begin{aligned}
& B\left(E_{0}(0,0)\right)=\left\{\left(x_{0}, y_{0}\right): 0 \leq x_{0}<\bar{x}, 0 \leq y_{0}<\bar{y}\right\}, \\
& B\left(E_{\bar{x}}(\bar{x}, 0)\right)=\left\{\left(x_{0}, y_{0}\right): \bar{x} \leq x_{0}<\infty, 0 \leq y_{0}<\bar{y}\right\}, \\
& B\left(E_{\bar{y}}(0, \bar{y})\right)=\left\{\left(x_{0}, y_{0}\right): 0 \leq x_{0}<\bar{x}, \bar{y} \leq y_{0}<\infty\right\}, \\
& B(E)=\left\{\left(x_{0}, y_{0}\right): \bar{x} \leq x_{0}, \bar{y} \leq y_{0}\right\} .
\end{aligned}
$$

(h) If $a=2, b>2$, then the basins of attraction of the equilibrium points are given as

$$
\begin{aligned}
& B\left(E_{0}(0,0)\right)=\left\{\left(x_{0}, y_{0}\right): 0 \leq x_{0}<\bar{x}, 0 \leq y_{0}<\bar{y}_{-}\right\}, \\
& B\left(E_{\bar{x}}(\bar{x}, 0)\right)=\left\{\left(x_{0}, y_{0}\right): \bar{x} \leq x_{0}<\infty, 0 \leq y_{0}<\bar{y}_{-}\right\}, \\
& B\left(E_{\bar{y}_{+}}\right)=\left\{\left(x_{0}, y_{0}\right): 0 \leq x_{0}<\bar{x}, \bar{y}_{-} \leq y_{0}<\infty\right\}, \\
& B\left(E_{+}\left(\bar{x}, \bar{y}_{+}\right)\right)=\left\{\left(x_{0}, y_{0}\right): \bar{x} \leq x_{0}, \bar{y}_{-}<y_{0}\right\} .
\end{aligned}
$$

(i) Case $a>2, b=2$, is symmetric to the case $a=2, b>2$.

Two species can interact in several different ways through competition, cooperation, or predator-prey interactions. For each of these interactions, we obtain variations of system (5), all of which may require a different mathematical analysis.

The following coupled system is a variation of system (5) that exhibits competitive interactions:

$$
\begin{aligned}
& x_{n+1}=\frac{a x_{n}^{2}}{1+x_{n}^{2}+c y_{n}}, \\
& y_{n+1}=\frac{b y_{n}^{2}}{1+y_{n}^{2}+d x_{n}}, \quad n=0,1, \ldots,
\end{aligned}
$$

where $a, b, c, d>0$. This system will be considered in the remainder of this paper. We will show that system (6) has similar but more complex dynamics than system (5). We will see that like system (5) the coupled system (6) may possess one, three, five, seven or nine 
equilibrium points in the hyperbolic case and two, four, six or eight equilibrium points in the non-hyperbolic case. In each of these cases we will show that the Allee effect is present and we will precisely describe the basins of attraction of all equilibrium points. We will show that the boundaries of the basins of attraction of the equilibrium points are the global stable manifolds of the saddle or the non-hyperbolic equilibrium points. See [9-15] for related results. An interesting feature of our results is that the size of the basin of attraction of the zero equilibrium decreases as a function of the number of the equilibrium points. The biological interpretation of our results is given in $[16,17]$ and a similar system is treated in [18]. However, no details of the proofs are provided in [16, 17] and non-hyperbolic cases were not treated there. Here we give the detailed proofs of all dynamic scenarios and provide the explicit algebraic conditions for the existence of one through nine equilibrium points, which was also missing in [16, 17]. A specific feature of our results is that no equilibrium point in the interior of the first quadrant is computable and so our analysis is based on the geometry of the equilibrium curves.

\section{Preliminaries}

Our proofs use some recent general results for competitive systems of difference equations of the form

$$
\left\{\begin{array}{l}
x_{n+1}=f\left(x_{n}, y_{n}\right) \\
y_{n+1}=g\left(x_{n}, y_{n}\right)
\end{array}\right.
$$

where $f$ and $g$ are continuous functions and $f(x, y)$ is non-decreasing in $x$ and nonincreasing in $y$ and $g(x, y)$ is non-increasing in $x$ and non-decreasing in $y$ in some domain $A$.

Competitive systems of the form (7) were studied by many authors in $[14,15,19-37]$ and others.

Here we give some basic notions as regards monotonic maps in a plane.

We define a partial order $\preceq_{\mathrm{se}}$ on $\mathbf{R}^{2}$ (the so-called southeast ordering) so that the positive cone is the fourth quadrant, i.e., this partial order is defined by

$$
\left(\begin{array}{l}
x^{1} \\
y^{1}
\end{array}\right) \preceq_{\text {se }}\left(\begin{array}{l}
x^{2} \\
y^{2}
\end{array}\right) \Leftrightarrow\left\{\begin{array}{l}
x^{1} \leq x^{2}, \\
y^{1} \geq y^{2} .
\end{array}\right.
$$

Similarly, we define northeast ordering as

$$
\left(\begin{array}{l}
x^{1} \\
y^{1}
\end{array}\right) \preceq_{\text {ne }}\left(\begin{array}{l}
x^{2} \\
y^{2}
\end{array}\right) \Leftrightarrow\left\{\begin{array}{l}
x^{1} \leq x^{2}, \\
y^{1} \leq y^{2} .
\end{array}\right.
$$

A map $F$ is called competitive if it is non-decreasing with respect to $\preceq_{\text {se }}$, that is, if the following holds:

$$
\left(\begin{array}{l}
x^{1} \\
y^{1}
\end{array}\right) \preceq\left(\begin{array}{l}
x^{2} \\
y^{2}
\end{array}\right) \Rightarrow F\left(\begin{array}{l}
x^{1} \\
y^{1}
\end{array}\right) \preceq F\left(\begin{array}{l}
x^{2} \\
y^{2}
\end{array}\right) .
$$

For each $\mathbf{v}=\left(v^{1}, v^{2}\right) \in \mathbf{R}_{+}^{2}$, define $\mathcal{Q}_{i}(\mathbf{v})$ for $i=1, \ldots, 4$ to be the usual four quadrants based at $v$ and numbered in a counterclock-wise direction, e.g., $\mathcal{Q}_{1}(\mathbf{v})=\left\{(x, y) \in \mathbf{R}_{+}^{2}: v^{1} \leq x\right.$, $\left.v^{2} \leq y\right\}$. 
For $S \subset R_{+}^{2}$ let $S^{\circ}$ denote the interior of $S$.

The following definition is from [35].

Definition 1 Let $R$ be a nonempty subset of $\mathbb{R}^{2}$. A competitive map $T: R \rightarrow R$ is said to satisfy condition $(O+)$ if for every $x, y$ in $R, T(x) \preceq_{\mathrm{ne}} T(y)$ implies $x \preceq_{\mathrm{ne}} y$, and $T$ is said to satisfy condition (O-) if for every $x, y$ in $R, T(x) \preceq_{\text {ne }} T(y)$ implies $y \preceq_{\text {ne }} x$.

The following theorem was proved by de Mottoni-Schiaffino [38] for the Poincaré map of a periodic competitive Lotka-Volterra system of differential equations. Smith generalized the proof to competitive and cooperative maps [34,39].

Theorem 4 Let $R$ be a nonempty subset of $\mathbb{R}^{2}$. If $T$ is a competitive map for which $\left(O_{+}\right)$ holds then for all $x \in R,\left\{T^{n}(x)\right\}$ is eventually component-wise monotone. If the orbit of $x$ has compact closure, then it converges to a fixed point of $T$. If instead $\left(O_{-}\right)$holds, then for all $x \in R,\left\{T^{2 n}\right\}$ is eventually component-wise monotone. If the orbit of $x$ has compact closure in $R$, then its omega limit set is either a period-two orbit or a fixed point.

It is well known that a stable period-two orbit and a stable fixed point may coexist; see Hess [40].

A non-hyperbolic equilibrium point $E$ of a competitive or cooperative map $T$ is called non-hyperbolic point of stable type (resp. of unstable type) if the second characteristic value of the Jacobian matrix $J_{T}(E)$ is in interval $(-1,1)$ (resp. outside of interval $[-1,1]$ ).

The following result is from [35], with the domain of the map specialized to be the Cartesian product of intervals of real numbers. It gives a sufficient condition for conditions $\left(\mathrm{O}_{+}\right)$ and $(O-)$.

Theorem 5 Let $R \subset \mathbb{R}^{2}$ be the Cartesian product of two intervals in $\mathbb{R}$. Let $T: R \rightarrow R$ be a $C^{\prime}$ competitive map. If $T$ is injective and $\operatorname{det} J_{T}(x)>0$ for all $x \in R$ then $T$ satisfies $(O+)$. If $T$ is injective and $\operatorname{det} J_{T}(x)<0$ for all $x \in R$ then $T$ satisfies $(O-)$.

Theorems 4 and 5 are quite applicable as we have shown in [41], in the case of competitive systems in the plane consisting of linear fractional equations.

The following result is from [13], which generalizes the corresponding result for hyperbolic case from [30]. Related results have been obtained by Smith in [34].

Theorem 6 Let $\mathcal{R}$ be a rectangular subset of $\mathbb{R}^{2}$ and let $T$ be a competitive map on $\mathcal{R}$. Let $\bar{x} \in \mathcal{R}$ be a fixed point of $T$ such that $\left(\mathcal{Q}_{1}(\bar{x}) \cup \mathcal{Q}_{3}(\bar{x})\right) \cap \mathcal{R}$ has nonempty interior (i.e., $\bar{x}$ is not the NW or $S E$ vertex of $\mathcal{R}$ ).

Suppose that the following statements are true.

(a) The map $T$ is strongly competitive on $\operatorname{int}\left(\left(\mathcal{Q}_{1}(\bar{x}) \cup \mathcal{Q}_{3}(\bar{x})\right) \cap \mathcal{R}\right)$.

(b) $T$ is $C^{2}$ on a relative neighborhood of $\bar{x}$.

(c) The Jacobian of $T$ at $\bar{x}$ has real eigenvalues $\lambda$, $\mu$ such that $|\lambda|<\mu$, where $\lambda$ is stable and the eigenspace $E^{\lambda}$ associated with $\lambda$ is not a coordinate axis.

(d) Either $\lambda \geq 0$ and

$$
T(x) \neq \bar{x} \quad \text { and } \quad T(x) \neq x \quad \text { for all } x \in \operatorname{int}\left(\left(\mathcal{Q}_{1}(\bar{x}) \cup \mathcal{Q}_{3}(\bar{x})\right) \cap \mathcal{R}\right),
$$


or $\lambda<0$ and

$$
T^{2}(x) \neq x \quad \text { for all } x \in \operatorname{int}\left(\left(\mathcal{Q}_{1}(\bar{x}) \cup \mathcal{Q}_{3}(\bar{x})\right) \cap \mathcal{R}\right) \text {. }
$$

Then there exists a curve $\mathcal{C}$ in $\mathcal{R}$ such that:

(i) $\mathcal{C}$ is invariant and a subset of $\mathcal{W}^{s}(\bar{x})$.

(ii) The endpoints of $\mathcal{C}$ lie on $\partial \mathcal{R}$.

(iii) $\bar{x} \in \mathcal{C}$.

(iv) $\mathcal{C}$ the graph of a strictly increasing continuous function of the first variable.

(v) $\mathcal{C}$ is differentiable at $\bar{x}$ if $\bar{x} \in \operatorname{int}(\mathcal{R})$ or one sided differentiable if $\bar{x} \in \partial \mathcal{R}$, and in all cases $\mathcal{C}$ is tangential to $E^{\lambda}$ at $\bar{x}$.

(vi) $\mathcal{C}$ separates $\mathcal{R}$ into two connected components, namely

$$
\mathcal{W}_{-}:=\{x \in \mathcal{R}: \exists y \in \mathcal{C} \text { with } x \preceq y\}
$$

and

$$
\mathcal{W}_{+}:=\{x \in \mathcal{R}: \exists y \in \mathcal{C} \text { with } y \preceq x\}
$$

(vii) $\mathcal{W}_{-}$is invariant, and $\operatorname{dist}\left(T^{n}(x), \mathcal{Q}_{2}(\bar{x})\right) \rightarrow 0$ as $n \rightarrow \infty$ for every $x \in \mathcal{W}_{-}$.

(viii) $\mathcal{W}_{+}$is invariant, and $\operatorname{dist}\left(T^{n}(x), \mathcal{Q}_{4}(\bar{x})\right) \rightarrow 0$ as $n \rightarrow \infty$ for every $x \in \mathcal{W}_{+}$.

The next results from [42] give the existence and uniqueness of invariant curves emanating from a non-hyperbolic point of unstable type, that is, a non-hyperbolic point where the second eigenvalue is outside the interval $[-1,1]$. See also [43].

Theorem 7 Let $\mathcal{R}=\left(a_{1}, a_{2}\right) \times\left(b_{1}, b_{2}\right)$, and let $T: \mathcal{R} \rightarrow \mathcal{R}$ be a strongly competitive map with a unique fixed point $\overline{\mathbf{x}} \in \mathcal{R}$, and such that $T$ is continuously differentiable in a neighborhood of $\overline{\mathbf{x}}$. Assume further that at the point $\overline{\mathbf{x}}$ the map $T$ has associated characteristic values $\mu$ and $\nu$ satisfying $1<\mu$ and $-\mu<v<\mu$.

Then there exist curves $\mathcal{C}_{1}, \mathcal{C}_{2}$ in $\mathcal{R}$ and there exist $\mathbf{p}_{1}, \mathbf{p}_{2} \in \partial \mathcal{R}$ with $\mathbf{p}_{1} \ll_{\text {se }} \overline{\mathbf{x}} \ll_{\text {se }} \mathbf{p}_{2}$ such that:

(i) For $\ell=1,2, \mathcal{C}_{\ell}$ is invariant, northeast strongly linearly ordered, such that $\overline{\mathbf{x}} \in \mathcal{C}_{\ell}$ and $\mathcal{C}_{\ell} \subset \mathcal{Q}_{3}(\overline{\mathbf{x}}) \cup \mathcal{Q}_{1}(\overline{\mathbf{x}})$; the endpoints $\mathbf{q}_{\ell}, \mathbf{r}_{\ell}$ of $\mathcal{C}_{\ell}$, where $\mathbf{q}_{\ell} \preceq_{\mathrm{ne}} \mathbf{r}_{\ell}$, belong to the boundary of $\mathcal{R}$. For $\ell, j \in\{1,2\}$ with $\ell \neq j, \mathcal{C}_{\ell}$ is a subset of the closure of one of the components of $\mathcal{R} \backslash \mathcal{C}_{j}$. Both $\mathcal{C}_{1}$ and $\mathcal{C}_{2}$ are tangential at $\overline{\mathbf{x}}$ to the eigenspace associated with $v$.

(ii) For $\ell=1,2$, let $B_{\ell}$ be the component of $\mathcal{R} \backslash \mathcal{C}_{\ell}$ whose closure contains $\mathbf{p}_{\ell}$. Then $B_{\ell}$ is invariant. Also, for $\mathbf{x} \in B_{1}, T^{n}(\mathbf{x})$ accumulates on $\mathcal{Q}_{2}\left(\mathbf{p}_{1}\right) \cap \partial \mathcal{R}$, and for $x \in B_{2}$, $T^{n}(\mathbf{x})$ accumulates on $\mathcal{Q}_{4}\left(\mathbf{p}_{2}\right) \cap \partial \mathcal{R}$.

(iii) Let $\mathcal{D}_{1}:=\mathcal{Q}_{1}(\overline{\mathbf{x}}) \cap \mathcal{R} \backslash\left(\mathcal{B}_{1} \cup \mathcal{B}_{2}\right)$ and $\mathcal{D}_{2}:=\mathcal{Q}_{3}(\overline{\mathbf{x}}) \cap \mathcal{R} \backslash\left(\mathcal{B}_{1} \cup \mathcal{B}_{2}\right)$. Then $\mathcal{D}_{1} \cup \mathcal{D}_{2}$ is invariant.

Corollary 1 Let a map $T$ with fixed point $\overline{\mathbf{x}}$ be as in Theorem 7. Let $\mathcal{D}_{1}, \mathcal{D}_{2}$ be the sets as in Theorem 7. If $T$ satisfies $(O+)$, then for $\ell=1,2, \mathcal{D}_{\ell}$ is invariant, and for every $\mathbf{x} \in \mathcal{D}_{\ell}$, the iterates $T^{n}(\mathbf{x})$ converge to $\overline{\mathbf{x}}$ or to a point of $\partial \mathcal{R}$. If $T$ satisfies $(O-)$, then $T\left(\mathcal{D}_{1}\right) \subset \mathcal{D}_{2}$ and $T\left(\mathcal{D}_{2}\right) \subset \mathcal{D}_{1}$. For every $\mathbf{x} \in \mathcal{D}_{1} \cup \mathcal{D}_{2}$, the iterates $T^{n}(\mathbf{x})$ either converge to $\overline{\mathbf{x}}$, or converge to a period-two point, or to a point of $\partial \mathcal{R}$. 


\section{Main results}

The main results of this paper depend on the number of interior equilibrium points of system (6). So first we give the explicit algebraic conditions in terms of the parameters for system (6) to have zero-five interior equilibrium points. Next, we present the local stability analysis of the equilibrium points and then the results on the global dynamics. It is interesting to note that the local stability analysis is the most difficult part of our analysis.

\subsection{Equilibrium points}

The equilibrium points of system (6) satisfy the following system of equations:

$$
\begin{aligned}
& \bar{x}=\frac{a \bar{x}^{2}}{1+\bar{x}^{2}+c \bar{y}}, \\
& \bar{y}=\frac{b \bar{y}^{2}}{1+\bar{y}^{2}+d \bar{x}}, \quad n=0,1, \ldots .
\end{aligned}
$$

All solutions of system (11) with at least one zero component are given as $E_{0}(0,0), E_{\bar{x}}(\bar{x}, 0)$ where $\bar{x}=1, E_{\bar{y}}(0, \bar{y})$ where $\bar{y}=1, E_{\bar{x}_{ \pm}}\left(0, \bar{x}_{ \pm}\right)$where $\bar{x}_{ \pm}=\frac{a \pm \sqrt{a^{2}-4}}{2}$, and $E_{\bar{y}_{ \pm}}\left(0, \bar{y}_{ \pm}\right)$where $\bar{y}_{ \pm}=\frac{b \pm \sqrt{b^{2}-4}}{2} \cdot E_{0}(0,0)$ exists in all cases. $E_{\bar{x}}(\bar{x}, 0)$ and $E_{\bar{y}}(0, \bar{y})$ exist when $a=2$ and $b=2$, respectively. $E_{\bar{x}_{ \pm}}\left(0, \bar{x}_{ \pm}\right)$and $E_{\bar{y}_{ \pm}}\left(0, \overline{y_{ \pm}}\right)$exist when $a>2$ and $b>2$, respectively.

The equilibrium points with strictly positive coordinates satisfy the following system of equations:

$$
\begin{aligned}
& -a x+c y+x^{2}+1=0, \\
& -b y+d x+y^{2}+1=0 .
\end{aligned}
$$

From (12) one can see that all positive solutions of system (12) satisfy the quartic equation:

$$
x^{4}-2 a x^{3}+x^{2}\left(a^{2}+b c+2\right)+x\left(c^{2} d-a(b c+2)\right)+b c+c^{2}+1=0 .
$$

Lemma 1 Let

$$
\begin{aligned}
\Delta_{3}= & -4 a\left(b^{2}-4\right) d\left(-4\left(a^{2}-4\right) b c+\left(a^{2}-4\right)^{2}-2\left(b^{2}-12\right) c^{2}\right) \\
& -4 a c^{2} d^{3}\left(a^{2}-18(b c+2)\right)+2 d^{2}\left(4 a^{4}(b c+2)-a^{2}(c(b(31 b c+64)-60 c)+64)\right. \\
& \left.-2(b c+2)\left(\left(b^{2}-36\right) c^{2}-32 b c-32\right)\right) \\
& +\left(b^{2}-4\right)^{2}\left(a^{4}-4 a^{2}(b c+2)+16(c(b+c)+1)\right)-27 c^{4} d^{4}, \\
\Delta_{2}= & \left(b^{2}-4\right)\left(a^{2}-2 b c-4\right)-2 a d\left(a^{2}-2 b c-4\right)-9 c^{2} d^{2}
\end{aligned}
$$

and

$$
\Delta_{1}=a^{2}-2 b c-4
$$

Then the following holds:

(a) If $\Delta_{3}>0, \Delta_{2}>0$, and $\Delta_{1}>0$, then (13) has four simple real roots.

(b) If $\Delta_{3}>0$ and $\Delta_{2} \leq 0 \vee\left(\Delta_{2}>0 \wedge \Delta_{1} \leq 0\right)$ then (13) has no real roots.

(c) If $\Delta_{3}<0$ then (13) has two simple real roots. 
(d) If $\Delta_{3}=0$ and $\Delta_{2}<0$ then (13) has one real double root.

(e) If $\Delta_{3}=0$ and $\Delta_{2}>0$ then (13) has two real simple roots and one real double root.

(f) If $\Delta_{3}=0, \Delta_{2}=0$ and $\Delta_{1}>0$ then (13) has two real double roots.

(g) If $\Delta_{3}=0, \Delta_{2}=0$ and $\Delta_{1}<0$ then (13) has no real roots.

(h) If $\Delta_{3}=0, \Delta_{2}=0$ and $\Delta_{1}=0$ then (13) has one real root of multiplicity four.

Proof The discrimination matrix [44] of $\tilde{f}$ and $\tilde{f}^{\prime}$ is given by

$\operatorname{Discr}(\tilde{f})$

$$
=\left(\begin{array}{cccccccc}
1 & -2 a & a^{2}+b c+2 & c^{2} d-a(b c+2) & c^{2}+b c+1 & 0 & 0 & 0 \\
0 & 4 & -6 a & 2\left(a^{2}+b c+2\right) & c^{2} d-a(b c+2) & 0 & 0 & 0 \\
0 & 1 & -2 a & a^{2}+b c+2 & c^{2} d-a(b c+2) & c^{2}+b c+1 & 0 & 0 \\
0 & 0 & 4 & -6 a & 2\left(a^{2}+b c+2\right) & c^{2} d-a(b c+2) & 0 & 0 \\
0 & 0 & 1 & -2 a & a^{2}+b c+2 & c^{2} d-a(b c+2) & c^{2}+b c+1 & 0 \\
0 & 0 & 0 & 4 & -6 a & 2\left(a^{2}+b c+2\right) & c^{2} d-a(b c+2) & 0 \\
0 & 0 & 0 & 1 & -2 a & a^{2}+b c+2 & c^{2} d-a(b c+2) & c^{2}+b c+1 \\
0 & 0 & 0 & 0 & 4 & -6 a & 2\left(a^{2}+b c+2\right) & c^{2} d-a(b c+2)
\end{array}\right),
$$

where

$$
\tilde{f}(x)=x^{4}-2 a x^{3}+x^{2}\left(a^{2}+b c+2\right)+x\left(c^{2} d-a(b c+2)\right)+b c+c^{2}+1 .
$$

Let $D_{k}$ denote the determinant of the submatrix of $\operatorname{Discr}(\tilde{f})$, formed by the first $2 k$ rows and the first $2 k$ columns, for $k=1,2,3,4$. So, by straightforward calculation one can see that $D_{1}=4, D_{2}=4 \Delta_{1}, D_{3}=4 c^{2} \Delta_{2}$, and $D_{4}=c^{4} \Delta_{3}$. The rest of the proof follows in view of [44, Theorem 1].

\subsection{Local stability of equilibrium points}

Geometrically the solutions of system (12) are intersections of two orthogonal parabolas that satisfy the equations

$$
\begin{aligned}
& y=-\frac{1}{c}\left(x-\frac{a}{2}\right)^{2}+\frac{a^{2}-4}{4 c}, \\
& x=-\frac{1}{d}\left(y-\frac{b}{2}\right)^{2}+\frac{b^{2}-4}{4 d},
\end{aligned}
$$

with respective vertices $\left(\frac{a}{2}, \frac{a^{2}-4}{4 c}\right)$ and $\left(\frac{b^{2}-4}{4 d}, \frac{b}{2}\right)$. See Figure 2 .

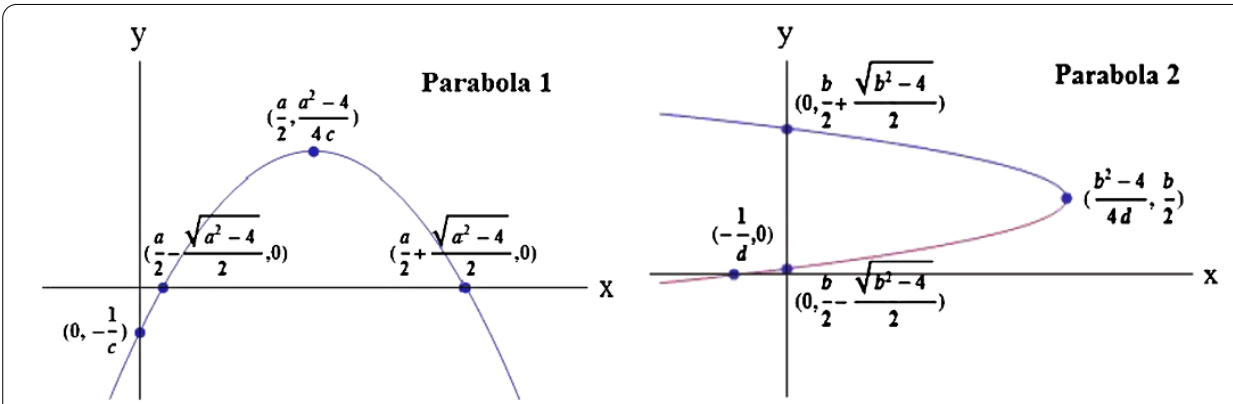

Figure 2 Equilibrium curves. 
Consequently when $a>2$ and $b>2$, in addition to the five equilibrium points on the axes, system (6) may have one, two, three or four positive equilibrium points. We will refer to these equilibrium points as $E_{\mathrm{SW}}(\bar{x}, \bar{y})$ (southwest), $E_{\mathrm{SE}}(\bar{x}, \bar{y})$ (southeast), $E_{\mathrm{NW}}(\bar{x}, \bar{y})$ (northwest), and $E_{\mathrm{NE}}(\bar{x}, \bar{y})$ (northeast) where

$$
E_{\mathrm{NW}} \preceq_{\mathrm{se}} E_{\mathrm{NE}} \preceq_{\mathrm{se}} E_{\mathrm{SE}}, \quad E_{\mathrm{SW}} \preceq_{\mathrm{ne}} E_{\mathrm{NW}} .
$$

When a positive equilibrium point is non-hyperbolic we will refer to it as $E_{N}(\bar{x}, \bar{y})$.

The map associated with system (6) has the form

$$
T\left(\begin{array}{l}
x \\
y
\end{array}\right)=\left(\begin{array}{c}
\frac{a x^{2}}{1+x^{2}+c y} \\
\frac{b y^{2}}{1+y^{2}+d x}
\end{array}\right) .
$$

The Jacobian matrix of $T$ is

$$
J_{T}(x, y)=\left(\begin{array}{cc}
\frac{2 a x(c y+1)}{\left(x^{2}+c y+1\right)^{2}} & -\frac{a c x^{2}}{\left(x^{2}+c y+1\right)^{2}} \\
-\frac{b d y^{2}}{\left(y^{2}+d x+1\right)^{2}} & \frac{2 b y(d x+1)}{\left(y^{2}+d x+1\right)^{2}}
\end{array}\right) .
$$

The Jacobian matrix of $T$ evaluated at an equilibrium $E(\bar{x}, \bar{y})$ with positive coordinates has the form

$$
J_{T}(\bar{x}, \bar{y})=\left(\begin{array}{cc}
\frac{2(c \bar{y}+1)}{a \bar{x}} & -\frac{c}{a} \\
-\frac{d}{b} & \frac{2(d \bar{x}+1)}{b \bar{y}}
\end{array}\right) .
$$

The determinant and trace of (19) are

$$
\begin{aligned}
& \operatorname{det} J_{T}(\bar{x}, \bar{y})=\frac{2(c \bar{y}+1)}{a \bar{x}} \frac{2(d+1)}{b \bar{y}}-\frac{d}{b} \frac{c}{a}=\frac{4 c \bar{y}+4 d \bar{x}+3 c d \bar{x} \bar{y}+4}{a \bar{x} b \bar{y}}, \\
& \operatorname{tr} J_{T}(\bar{x}, \bar{y})=\frac{2(c \bar{y}+1)}{a \bar{x}}+\frac{2(d \bar{x}+1)}{b \bar{y}} .
\end{aligned}
$$

It is worth noting that $\operatorname{det} J_{T}(\bar{x}, \bar{y})$ and $\operatorname{tr} J_{T}(\bar{x}, \bar{y})$ of (19) are both positive.

Using the equilibrium condition (12), we may rewrite the determinant and trace as

$$
\begin{aligned}
& \operatorname{det} J_{T}(\bar{x}, \bar{y})=\frac{2(a-\bar{x})}{a} \frac{2(b-\bar{y})}{b}-\frac{d}{b} \frac{c}{a}, \\
& \operatorname{tr} J_{T}(\bar{x}, \bar{y})=\frac{2(a-\bar{x})}{a}+\frac{2(b-\bar{y})}{b} .
\end{aligned}
$$

We will use both (20) and (21) in our proofs to follow.

The characteristic equation of the matrix (19) is

$$
\lambda^{2}-\operatorname{tr} J_{T}(\bar{x}, \bar{y}) \lambda+\operatorname{det} J_{T}(\bar{x}, \bar{y})=0
$$

of which the solutions are the eigenvalues

$$
\begin{aligned}
& \lambda=\frac{\operatorname{tr} J_{T}(\bar{x}, \bar{y})-\sqrt{\left(\operatorname{tr} J_{T}(\bar{x}, \bar{y})\right)^{2}-4 \operatorname{det} J_{T}(\bar{x}, \bar{y})}}{2}, \\
& \mu=\frac{\operatorname{tr} J_{T}(\bar{x}, \bar{y})+\sqrt{\left(\operatorname{tr} J_{T}(\bar{x}, \bar{y})\right)^{2}-4 \operatorname{det} J_{T}(\bar{x}, \bar{y})}}{2} .
\end{aligned}
$$


The eigenvalues of (19) are therefore

$$
\begin{aligned}
& \lambda=\frac{\left(\frac{2(c \bar{y}+1)}{a \bar{x}}+\frac{2(d \bar{x}+1)}{b \bar{y}}\right)-\sqrt{\left(\frac{2(c \bar{y}+1)}{a \bar{x}}-\frac{2(d \bar{x}+1)}{b \bar{y}}\right)^{2}+4 \frac{d c}{b a}}}{2}, \\
& \mu=\frac{\left(\frac{2(c \bar{y}+1)}{a \bar{x}}+\frac{2(d \bar{x}+1)}{b \bar{y}}\right)+\sqrt{\left(\frac{2(c \bar{y}+1)}{a \bar{x}}-\frac{2(d \bar{x}+1)}{b \bar{y}}\right)^{2}+4 \frac{d c}{b a}}}{2},
\end{aligned}
$$

with corresponding eigenvectors

$$
\begin{aligned}
& E_{\lambda}=\left(-\frac{b}{d}\left(\frac{(c \bar{y}+1)}{a \bar{x}}-\frac{(d \bar{x}+1)}{b \bar{y}}-\frac{\sqrt{\left(\frac{2(c \bar{y}+1)}{a \bar{x}}-\frac{2(d \bar{x}+1)}{b \bar{y}}\right)^{2}+4 \frac{d c}{b a}}}{2}\right), 1\right), \\
& E_{\mu}=\left(-\frac{b}{d}\left(\frac{(c \bar{y}+1)}{a \bar{x}}-\frac{(d \bar{x}+1)}{b \bar{y}}+\frac{\sqrt{\left(\frac{2(c \bar{y}+1)}{a \bar{x}}-\frac{2(d \bar{x}+1)}{b \bar{y}}\right)^{2}+4 \frac{d c}{b a}}}{2}\right), 1\right) .
\end{aligned}
$$

Using the equilibrium condition (12), we may rewrite the eigenvalues and eigenvectors as

$$
\begin{aligned}
& \lambda=\frac{\left(\frac{2(a-\bar{x})}{a}+\frac{2(b-\bar{y})}{b}\right)-\sqrt{\left(\frac{2(a-\bar{x})}{a}-\frac{2(b-\bar{y})}{b}\right)^{2}+4 \frac{d c}{b a}}}{2}, \\
& \mu=\frac{\left(\frac{2(a-\bar{x})}{a}+\frac{2(b-\bar{y})}{b}\right)+\sqrt{\left(\frac{2(a-\bar{x})}{a}-\frac{2(b-\bar{y})}{b}\right)^{2}+4 \frac{d c}{b a}}}{2} \\
& E_{\lambda}=\left(-\frac{b}{d}\left(\frac{(a-\bar{x})}{a}-\frac{(b-\bar{y})}{b}-\frac{\sqrt{\left(\frac{2(a-\bar{x})}{a}-\frac{2(b-\bar{y})}{b}\right)^{2}+4 \frac{d c}{b a}}}{2}\right), 1\right), \\
& E_{\mu}=\left(-\frac{b}{d}\left(\frac{(a-\bar{x})}{a}-\frac{(b-\bar{y})}{b}+\frac{\sqrt{\left(\frac{2(a-\bar{x})}{a}-\frac{2(b-\bar{y})}{b}\right)^{2}+4 \frac{d c}{b a}}}{2}\right), 1\right) .
\end{aligned}
$$

We will now consider two lemmas that will be used to prove the local stability character of the positive equilibrium points of system (6). The nonzero coordinates, $(\bar{x}, \bar{y})$, of all equilibrium points will subsequently be designated with the subscripts: $r$ (repeller), $a$ (attractor), $s, s_{1}, s_{2}$ (saddle point), $n s_{1}, n s_{2}$ (non-hyperbolic of the stable type), and $n u$ (non-hyperbolic of the unstable type).

Lemma 2 The following conditions hold for the coordinates of the positive equilibrium points, $E(\bar{x}, \bar{y})$, of system (6).

(i) For $E_{\mathrm{SW}}\left(\bar{x}_{r}, \bar{y}_{r}\right)$

$$
\bar{x}<\frac{a}{2}<\frac{b^{2}-4}{4 d} \text { and } \bar{y}<\frac{b}{2}<\frac{a^{2}-4}{4 c} .
$$

(ii) For $E_{\mathrm{NW}}\left(\bar{x}_{s_{1}}, \bar{y}_{s_{1}}\right)$,

$$
\bar{x}<\frac{a}{2}<\frac{b^{2}-4}{4 d} \text { and } \frac{b}{2}<\bar{y}<\frac{a^{2}-4}{4 c} .
$$


(iii) For $E_{\mathrm{NE}}\left(\bar{x}_{a}, \bar{y}_{a}\right)$,

$$
\frac{a}{2}<\bar{x}<\frac{b^{2}-4}{4 d} \quad \text { and } \quad \frac{b}{2}<\bar{y}<\frac{a^{2}-4}{4 c} .
$$

(iv) For $E_{\mathrm{SE}}\left(\bar{x}_{s_{2}}, \bar{y}_{s_{2}}\right)$,

$$
\frac{a}{2}<\bar{x}<\frac{b^{2}-4}{4 d} \text { and } \bar{y}<\frac{b}{2}<\frac{a^{2}-4}{4 c} .
$$

(v) For $E_{N}\left(\bar{x}_{n s 1}, \bar{y}_{n s 1}\right)$ and $E_{N}\left(\bar{x}_{n s 2}, \bar{y}_{n s 2}\right)$,

$$
\frac{a}{2} \leq \bar{x}<\frac{b^{2}-4}{4 d} \quad \text { and } \quad \frac{b}{2}<\bar{y} \leq \frac{a^{2}-4}{4 c} .
$$

(vi) $\operatorname{For} E_{N}\left(\bar{x}_{n u}, \bar{y}_{n u}\right)$,

$$
\bar{x}<\frac{b^{2}-4}{4 d}<\frac{a}{2} \text { and } \bar{y}<\frac{a^{2}-4}{4 c}<\frac{b}{2} .
$$

Proof This is clear from the geometry. See Figure 3.

Lemma 3 The following conditions hold for the coordinates of the positive equilibrium points, $E(\bar{x}, \bar{y})$, of system (6).

(i) For $E_{\mathrm{SW}}\left(\bar{x}_{r}, \bar{y}_{r}\right)$ and $E_{\mathrm{NW}}\left(\bar{x}_{s_{1}}, \bar{y}_{s_{1}}\right)$,

$$
c d<(a-2 \bar{x})(b-2 \bar{y}) .
$$

(ii) For $E_{\mathrm{NE}}\left(\bar{x}_{a}, \bar{y}_{a}\right)$ and $E_{\mathrm{SE}}\left(\bar{x}_{s_{2}}, \bar{y}_{s_{2}}\right)$,

$$
c d>(a-2 \bar{x})(b-2 \bar{y}) .
$$

(iii) For $E_{N}\left(\bar{x}_{n s 1}, \bar{y}_{n s 1}\right), E_{N}\left(\bar{x}_{n s 2}, \bar{y}_{n s 2}\right)$, and $E_{N}\left(\bar{x}_{n u}, \bar{y}_{n u}\right)$,

$$
c d=(a-2 \bar{x})(b-2 \bar{y}) .
$$

Proof (i) Let $m_{P 1}$ be the slope of the tangent line to parabola $P_{1}$ at $E(\bar{x}, \bar{y})=E_{\mathrm{SW}}\left(\bar{x}_{r}, \bar{y}_{r}\right)$ and let $m_{P 2}$ be the slope of the tangent line to parabola $P_{2}$ at $E(\bar{x}, \bar{y})=E_{\mathrm{SW}}\left(\bar{x}_{r}, \bar{y}_{r}\right)$. It is clear from the geometry that

$$
m_{P 1}>m_{P 2}>0 .
$$

See Figure 3. It follows that

$$
\left.\frac{d y}{d x}\right|_{P_{1}}(\bar{x}, \bar{y})>\left.\frac{d x}{d y}\right|_{P_{2}}(\bar{x}, \bar{y})>0
$$

and in turn

$$
\frac{a-2 \bar{x}}{c}>\frac{d}{b-2 \bar{y}}>0 .
$$




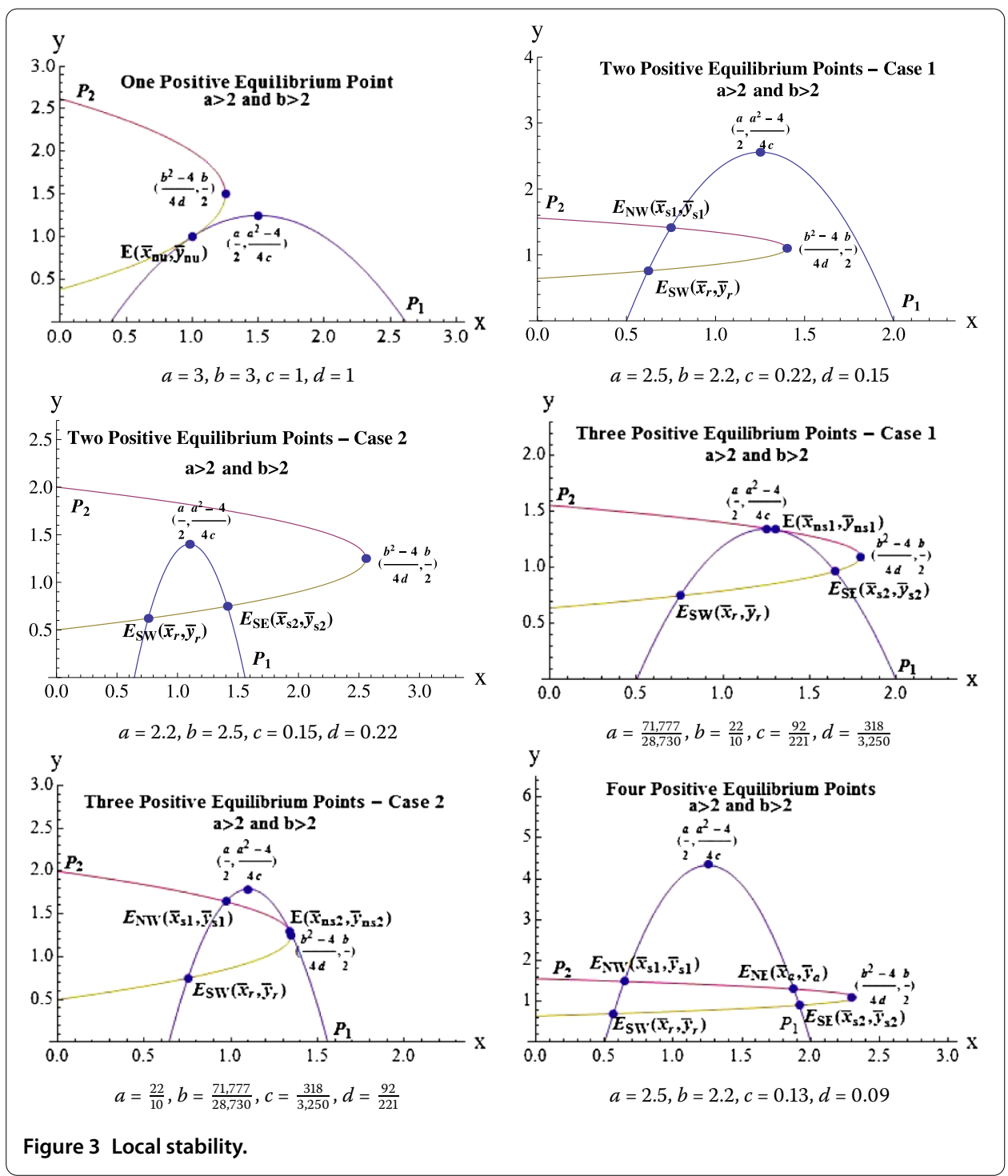

Therefore

$$
c d<(a-2 \bar{x})(b-2 \bar{y})
$$

The proofs for cases (ii) and (iii) are similar and will be omitted.

Theorem 8 The following conditions hold for the equilibrium points $E(\bar{x}, \bar{y})$ of system (6).

(i) $E_{0}(0,0)$ is locally asymptotically stable.

(ii) $E_{\bar{x}}\left(\bar{x}_{n s}, 0\right)$ and $E_{\bar{y}}\left(0, \bar{y}_{n s}\right)$ are non-hyperbolic of the stable type.

(iii) $E_{\bar{x}_{+}}\left(\bar{x}_{+a}, 0\right)$ is locally asymptotically stable and $E_{\bar{x}_{-}}\left(\bar{x}_{-s}, 0\right)$ is a saddle point.

(iv) $E_{\bar{y}_{+}}\left(0, \bar{y}_{+a}\right)$ is locally asymptotically stable and $E_{\bar{y}_{-}}\left(0, \bar{y}_{-s}\right)$ is a saddle point.

(v) $E_{\mathrm{SW}}\left(\bar{x}_{r}, \bar{y}_{r}\right)$ is a repeller.

(vi) $E_{\mathrm{NW}}\left(\bar{x}_{s_{1}}, \bar{y}_{s_{1}}\right)$ and $E_{\mathrm{SE}}\left(\bar{x}_{s_{2}}, \bar{y}_{s_{2}}\right)$ are saddle points.

(vii) $E_{\mathrm{NE}}\left(\bar{x}_{a}, \bar{y}_{a}\right)$ is locally asymptotically stable.

(viii) $E_{N}\left(\bar{x}_{n s 1}, \bar{y}_{n s 1}\right)$ and $E_{N}\left(\bar{x}_{n s 2}, \bar{y}_{n s 2}\right)$ are non-hyperbolic of the stable type. 
(ix) $E_{N}\left(\bar{x}_{n u}, \bar{y}_{n u}\right)$ is non-hyperbolic of the unstable type.

Proof (i) The eigenvalues of (18), evaluated at $E_{\bar{x}}(0,0)$, are $\lambda=0$ and $\mu=0$.

(ii) The eigenvalues of (18), evaluated at $E_{\bar{x}}\left(\bar{x}_{n s}, 0\right)$, are $\lambda=0$ and $\mu=1$ when $a=2$. The eigenvalues of (18), evaluated at $E_{\bar{y}}\left(0, \bar{y}_{n s}\right)$, are $\lambda=0$ and $\mu=1$ when $b=2$.

(iii) The eigenvalues of (18), evaluated at $E_{\bar{x}_{+}}\left(\bar{x}_{+a}, 0\right)$ and $E_{\bar{x}_{-}}\left(\bar{x}_{-s}, 0\right)$, respectively, are $\lambda=0$ and $\mu_{ \pm}=\frac{2 a\left(\frac{a \pm \sqrt{a^{2}-4}}{2}\right)}{\left(\frac{a \pm \sqrt{a^{2}-4}}{2}\right)^{4}+2\left(\frac{a \pm \sqrt{a^{2}-4}}{2}\right)^{2}+1}$ when $a>2$.

(a) Note that when $a>2$,

$$
\begin{aligned}
\mu_{+} & =\frac{2 a\left(\frac{a+\sqrt{a^{2}-4}}{2}\right)}{\left(\frac{a+\sqrt{a^{2}-4}}{2}\right)^{4}+2\left(\frac{a+\sqrt{a^{2}-4}}{2}\right)^{2}+1} \\
& =\frac{4 a+4 \sqrt{a^{2}-4}}{4 a+4 \sqrt{a^{2}-4}+a^{2} \sqrt{a^{2}-4}+\left(a^{2}-4\right)^{\frac{3}{2}}+2 a\left(a^{2}-4\right)} \\
& <\frac{4 a+4 \sqrt{a^{2}-4}}{4 a+4 \sqrt{a^{2}-4}}=1 .
\end{aligned}
$$

(b) Note that when $a>2$,

$$
\begin{aligned}
\mu_{-}= & \frac{2 a\left(\frac{a-\sqrt{a^{2}-4}}{2}\right)}{\left(\frac{a-\sqrt{a^{2}-4}}{2}\right)^{4}+2\left(\frac{a-\sqrt{a^{2}-4}}{2}\right)^{2}+1} \\
& =\frac{4 a-4 \sqrt{a^{2}-4}}{4 a-4 \sqrt{a^{2}-4}-a^{2} \sqrt{a^{2}-4}-\sqrt{\left(a^{2}-4\right)^{3}}+2 a^{3}-8 a} .
\end{aligned}
$$

It can be shown that

$$
-a^{2} \sqrt{a^{2}-4}-\sqrt{\left(a^{2}-4\right)^{3}}+2 a^{3}-8 a=2 a\left(a^{2}-4\right)\left(1-\sqrt{1+\frac{4}{a^{2}\left(a^{2}-4\right)}}\right)<0 .
$$

Therefore,

$$
\mu_{-}>\frac{4 a-4 \sqrt{a^{2}-4}}{4 a-4 \sqrt{a^{2}-4}}=1
$$

In both cases, the conclusion follows.

(iv) The eigenvalues of (18), evaluated at $E_{\bar{y}_{+}}\left(0, \bar{y}_{+a}\right)$ and $E_{\bar{y}_{-}}\left(0, \bar{y}_{-s}\right)$, respectively, are $\lambda=0$ and $\mu_{ \pm}=\frac{2 b\left(\frac{b \pm \sqrt{b^{2}-4}}{2}\right)}{\left(\frac{b \pm \sqrt{b^{2}-4}}{2}\right)^{4}+2\left(\frac{b \pm \sqrt{b^{2}-4}}{2}\right)^{2}+1}$ when $b>2$.

The proof of (iv) is similar to the proof of (iii) and will be omitted.

(v) We need to show that $\left|\operatorname{tr} J_{T}(\bar{x}, \bar{y})\right|<\left|1+\operatorname{det} J_{T}(\bar{x}, \bar{y})\right|$ and $\left|\operatorname{det} J_{T}(\bar{x}, \bar{y})\right|>1$ when $E(\bar{x}, \bar{y})=E_{\mathrm{SW}}\left(\bar{x}_{r}, \bar{y}_{r}\right)$. Since $\operatorname{tr} J_{T}(\bar{x}, \bar{y})$ and $\operatorname{det} J_{T}(\bar{x}, \bar{y})$ are both positive, our conditions become $\operatorname{tr} J_{T}(\bar{x}, \bar{y})<1+\operatorname{det} J_{T}(\bar{x}, \bar{y})$ and $\operatorname{det} J_{T}(\bar{x}, \bar{y})>1$. We will first show that $\operatorname{det} J_{T}(\bar{x}, \bar{y})>1$. By (34) we have

$$
\begin{aligned}
& \operatorname{det}\left(J_{T}(\bar{x}, \bar{y})\right)-1 \\
& =\frac{2(a-\bar{x})}{a} \frac{2(b-\bar{y})}{b}-\frac{d}{b} \frac{c}{a}-1
\end{aligned}
$$




$$
\begin{aligned}
& >\frac{2(a-\bar{x})}{a} \frac{2(b-\bar{y})}{b}-\frac{(a-2 \bar{x})(b-2 \bar{y})}{a b}-1 \\
& =2\left(1-\frac{\bar{y}}{b}-\frac{\bar{x}}{a}\right) .
\end{aligned}
$$

By (28) we have

$$
1-\frac{\bar{y}}{b}-\frac{\bar{x}}{a}>0 .
$$

Therefore $\operatorname{det}\left(J_{T}(\bar{x}, \bar{y})\right)>1$. We will next show that $\operatorname{tr}\left(J_{T}(\bar{x}, \bar{y})\right)<1+\operatorname{det}\left(J_{T}(\bar{x}, \bar{y})\right)$. By (34) we have

$$
\begin{aligned}
1 & +\operatorname{det}\left(J_{T}(\bar{x}, \bar{y})\right)-1 \\
& =1+\frac{2(a-\bar{x})}{a} \frac{2(b-\bar{y})}{b}-\frac{d}{b} \frac{c}{a}-1 \\
& >1+\frac{2(a-\bar{x})}{a} \frac{2(b-\bar{y})}{b}-\frac{(a-2 \bar{x})(b-2 \bar{y})}{a b}-1 \\
& =\operatorname{tr}\left(J_{T}(\bar{x}, \bar{y})\right)-1 .
\end{aligned}
$$

Therefore $\operatorname{tr}\left(J_{T}(\bar{x}, \bar{y})\right)<1+\operatorname{det}\left(J_{T}(\bar{x}, \bar{y})\right)$.

(vi) We need to show that $|\operatorname{tr} J(\bar{x}, \bar{y})|>\left|1+\operatorname{det} J_{T}(\bar{x}, \bar{y})\right|$ when $E(\bar{x}, \bar{y})=E_{\mathrm{NW}}\left(\bar{x}_{s_{1}}, \bar{y}_{s_{1}}\right)$. Since $\operatorname{tr} J_{T}(\bar{x}, \bar{y})$ and $\operatorname{det}_{T}(\bar{x}, \bar{y})$ are both positive, our condition becomes $\operatorname{tr} J_{T}(\bar{x}, \bar{y})>1+\operatorname{det} J_{T}(\bar{x}, \bar{y})$. By (34) we have

$$
\begin{aligned}
1 & +\operatorname{det}\left(J_{T}(\bar{x}, \bar{y})\right) \\
& =1+\frac{2(a-\bar{x})}{a} \frac{2(b-\bar{y})}{b}-\frac{d}{b} \frac{c}{a} \\
& <1+\frac{2(a-\bar{x})}{a} \frac{2(b-\bar{y})}{b}-\frac{(a-2 \bar{x})(b-2 \bar{y})}{a b} \\
& =\frac{2(a-\bar{x})}{a}+\frac{2(b-\bar{y})}{b} \\
& =\operatorname{tr}\left(J_{T}(\bar{x}, \bar{y})\right) .
\end{aligned}
$$

Therefore $\operatorname{tr} J_{T}(\bar{x}, \bar{y})>1+\operatorname{det} J_{T}(\bar{x}, \bar{y})$. The proof that $E_{\mathrm{SE}}\left(\bar{x}_{s_{2}}, \bar{y}_{s_{2}}\right)$ is a saddle point is similar and will be omitted.

(vii) We need to show that $\left|\operatorname{tr} J_{T}(\bar{x}, \bar{y})\right|<1+\operatorname{det} J_{T}(\bar{x}, \bar{y})$ and $\operatorname{det} J_{T}(\bar{x}, \bar{y})<1$ when $E(\bar{x}, \bar{y})=$ $E_{\mathrm{NE}}\left(\bar{x}_{a}, \bar{y}_{a}\right)$. Since $\operatorname{tr} J_{T}(\bar{x}, \bar{y})$ and $\operatorname{det} J_{T}(\bar{x}, \bar{y})$ are both positive, our conditions become $\operatorname{tr} J_{T}(\bar{x}, \bar{y})<1+\operatorname{det} J_{T}(\bar{x}, \bar{y})$ and $\operatorname{det} J_{T}(\bar{x}, \bar{y})<1$. We will first show that $\operatorname{det} J_{T}(\bar{x}, \bar{y})<1$. By (35) we have

$$
\operatorname{det}\left(J_{T}(\bar{x}, \bar{y})\right)-1=\frac{2(c \bar{y}+1)}{a \bar{x}} \frac{2(d \bar{x}+1)}{b \bar{y}}-\frac{d}{b} \frac{c}{a}-1 .
$$

By (30) we have

$$
\begin{aligned}
& \frac{2(c \bar{y}+1)}{a \bar{x}} \frac{2(d \bar{x}+1)}{b \bar{y}}-\frac{d}{b} \frac{c}{a}-1 \\
& <\frac{2\left(\frac{a^{2}}{4}\right)}{a \bar{x}} \frac{2\left(\frac{b^{2}}{4}\right)}{b \bar{y}}-\frac{d}{b} \frac{c}{a}-1=\frac{a b}{4 \bar{x} \bar{y}}-\frac{d}{b} \frac{c}{a}-1 .
\end{aligned}
$$


By (30) again we have

$$
\frac{a b}{4 \bar{x} \bar{y}}-\frac{d}{b} \frac{c}{a}-1<1-\frac{d}{b} \frac{c}{a}-1=-\frac{d}{b} \frac{c}{a}<0
$$

Therefore $\operatorname{det}\left(J_{T}(\bar{x}, \bar{y})\right)<1$. We will next show that $1+\operatorname{det}\left(J_{F}(\bar{x}, \bar{y})\right)>\operatorname{tr}\left(J_{F}(\bar{x}, \bar{y})\right)$.

By (35) we have

$$
\begin{aligned}
1 & +\operatorname{det}\left(J_{F}(\bar{x}, \bar{y})\right)-\operatorname{tr}\left(J_{F}(\bar{x}, \bar{y})\right) \\
& =1+\frac{2(a-\bar{x})}{a} \frac{2(b-\bar{y})}{b}-\frac{d}{b} \frac{c}{a}-\frac{2(a-\bar{x})}{a}-\frac{2(b-\bar{y})}{b} \\
& >1+\frac{2(a-\bar{x})}{a} \frac{2(b-\bar{y})}{b}-\frac{(a-2 \bar{x})(b-2 \bar{y})}{a b}-\frac{2(a-\bar{x})}{a}-\frac{2(b-\bar{y})}{b}=0 .
\end{aligned}
$$

Therefore $1+\operatorname{det}\left(J_{F}(\bar{x}, \bar{y})\right)>\operatorname{tr}\left(J_{F}(\bar{x}, \bar{y})\right)$.

(viii) By (26) and (36), we have

$$
\begin{aligned}
& \lambda=3-\frac{2 y}{b}-\frac{2 x}{a}, \\
& \mu=1 .
\end{aligned}
$$

By (32), we have $\lambda<1$. The conclusion follows.

(ix) The proof of (ix) is similar to the proof of (viii) and will be omitted.

\subsection{Global results}

In this section we combine the results from Sections 2 and 3.2 to prove the global results for system (6). First, we prove that the map $T$ which corresponds to system (6) is injective and it satisfies $(\mathrm{O}+)$.

Theorem 9 The map $T$ which corresponds to system (6) is injective.

Proof Indeed,

$$
T\left(\begin{array}{l}
x_{1} \\
y_{1}
\end{array}\right)=T\left(\begin{array}{l}
x_{2} \\
y_{2}
\end{array}\right) \Leftrightarrow\left(\begin{array}{c}
\frac{a x_{1}^{2}}{1+x_{1}^{2}+c y_{1}} \\
\frac{b y_{1}^{2}}{1+y_{1}^{2}+d x_{1}}
\end{array}\right)=\left(\begin{array}{c}
\frac{a x_{2}^{2}}{1+x_{2}^{2}+c y_{2}} \\
\frac{b y_{2}^{2}}{1+y_{2}^{2}+d x_{2}}
\end{array}\right),
$$

which is equivalent to

$$
\begin{aligned}
& x_{1}^{2}-x_{2}^{2}=c\left(x_{2}^{2} y_{1}-x_{1}^{2} y_{2}\right), \\
& y_{1}^{2}-y_{2}^{2}=d\left(y_{2}^{2} x_{1}-y_{1}^{2} x_{2}\right) .
\end{aligned}
$$

Now we will prove that $x_{1}=x_{2}$, which immediately implies $y_{1}=y_{2}$.

First, assume $x_{1}>x_{2}$. Then $x_{2}^{2} y_{1}-x_{1}^{2} y_{2}>0$ and $x_{2}^{2} y_{1}>x_{1}^{2} y_{2}$ which in view of (37) implies $y_{1}>y_{2}$. In view of (38), $y_{2}^{2} x_{1}-y_{1}^{2} x_{2}>0$, that is, $x_{1} y_{2} \cdot y_{2}>x_{2} y_{1} \cdot y_{1}$, which implies $x_{1} y_{2}>x_{2} y_{1}$ and $x_{1}^{2} y_{2}>x_{1} x_{2} y_{1}>x_{2}^{2} y_{1}$ and in view of (37) we obtain $x_{1}<x_{2}$, which is a contradiction. 
Second, assume $x_{1}<x_{2}$. Then $x_{2}^{2} y_{1}<x_{1}^{2} y_{2}$, which implies $y_{2}>y_{1}$ and $y_{2}^{2} x_{1}-y_{1}^{2} x_{2}<0$ and $x_{1} y_{2} \cdot y_{2}<x_{2} y_{1} \cdot y_{1}$, which is equivalent to $x_{1} y_{2}<x_{2} y_{1}$ and $x_{2}^{2} y_{1}>x_{1} x_{2} y_{1}>x_{1}^{2} y_{2}$. In view of (37) we obtain $x_{1}>x_{2}$, which is a contradiction.

Thus $x_{1}=x_{2}$ and $T$ is injective.

Theorem 10 The map $T$ which corresponds to system (6) satisfies (O+). All solutions of system (6) converge to an equilibrium point.

Proof Assume that

$$
T\left(\begin{array}{l}
x_{1} \\
y_{1}
\end{array}\right) \leq_{\mathrm{ne}} T\left(\begin{array}{l}
x_{2} \\
y_{2}
\end{array}\right) \Leftrightarrow\left(\begin{array}{c}
\frac{a x_{1}^{2}}{1+x_{1}^{2}+c y_{1}} \\
\frac{b y_{1}^{2}}{1+y_{1}^{2}+d x_{1}}
\end{array}\right) \leq\left(\begin{array}{c}
\frac{a x_{2}^{2}}{1+x_{2}^{2}+c y_{2}} \\
\frac{b y_{2}^{2}}{1+y_{2}^{2}+d x_{2}}
\end{array}\right) .
$$

The last inequality is equivalent to

$$
\begin{aligned}
& x_{1}^{2}-x_{2}^{2} \leq c\left(x_{2}^{2} y_{1}-x_{1}^{2} y_{2}\right), \\
& y_{1}^{2}-y_{2}^{2} \leq d\left(y_{2}^{2} x_{1}-y_{1}^{2} x_{2}\right) .
\end{aligned}
$$

First we prove that $x_{1} \leq x_{2}$. Otherwise $x_{1}>x_{2}$.

Then

$$
x_{2}^{2} y_{1}>x_{1}^{2} y_{2}
$$

which implies $y_{1}>y_{2} \Rightarrow y_{2}^{2} x_{1}-y_{1}^{2} x_{2}>0$, which is equivalent to $x_{1} y_{2} \cdot y_{2}-x_{2} y_{1} \cdot y_{1}>0$ and implies $x_{1} y_{2}>x_{2} y_{1}$, which in turn implies $x_{1}^{2} y_{2}>x_{1} x_{2} y_{1}>x_{2}^{2} y_{1}$, which contradicts (39). Consequently $x_{1} \leq x_{2}$.

Next we prove that $y_{1} \leq y_{2}$. Otherwise $y_{1}>y_{2}$.

Then $x_{1} y_{2}^{2}>x_{2} y_{1}^{2}$, which implies $x_{1}>x_{2}$, which is impossible in view of $x_{1} \leq x_{2}$.

Thus $\left(\begin{array}{l}x_{1} \\ y_{1}\end{array}\right) \leq$ ne $\left(\begin{array}{l}x_{2} \\ y_{2}\end{array}\right)$.

Thus we conclude that all solutions of (6) are eventually monotonic for all values of parameters. Furthermore it is clear that all solutions are bounded. Indeed every solution of (6) satisfies

$$
x_{n} \leq a, \quad y_{n} \leq b, \quad n=1,2, \ldots
$$

Consequently, all solutions converge to an equilibrium point.

Theorem 11 Assume that $a<2$ and $b<2$. Then the zero equilibrium of (6) is globally asymptotically stable.

Proof It follows immediately from Theorem 10.

\section{Theorem 12}

(a) If $a=2, b<2$, then system (6) has two equilibrium points, $E_{0}, E_{\bar{x}}$, where $E_{0}$ is locally asymptotically stable and $E_{\bar{x}}$ is non-hyperbolic of the stable type. The basins of 
attraction of the two equilibrium points are given as

$$
\begin{aligned}
& B\left(E_{0}(0,0)\right)=\left\{\left(x_{0}, y_{0}\right) \text { : points above } \mathcal{W}^{s}\left(E_{\bar{x}}\right)\right\}, \\
& B\left(E_{\bar{x}}\right)=\left\{\left(x_{0}, y_{0}\right) \text { : points below } \mathcal{W}^{s}\left(E_{\bar{x}}\right)\right\},
\end{aligned}
$$

where $\mathcal{W}^{s}(E)$ denotes the global stable manifold guaranteed by Theorem 6 .

(b) Similarly, if $a<2, b=2$, the basins of attraction of the equilibrium points are given as

$$
\begin{aligned}
& B\left(E_{0}(0,0)\right)=\left\{\left(x_{0}, y_{0}\right) \text { : points below } \mathcal{W}^{s}\left(E_{\bar{y}}\right)\right\}, \\
& B\left(E_{\bar{y}}\right)=\left\{\left(x_{0}, y_{0}\right) \text { : points above } \mathcal{W}^{s}\left(E_{\bar{y}}\right)\right\} .
\end{aligned}
$$

Proof We will present the proof of (a) since the proof of (b) uses analogous arguments. Local stability of the equilibrium points follows from Theorem 8 . Furthermore, the existence of the stable manifold $\mathcal{W}^{s}\left(E_{\bar{x}}\right)$ follows from Theorem 6 . By immediate checking one can see that if $x_{0}>\bar{x}$ then $T^{n}\left(x_{0}, 0\right) \rightarrow E_{x}$ as $n \rightarrow \infty$ and if $x_{0}<\bar{x}$ then $T^{n}\left(x_{0}, 0\right) \rightarrow E_{0}$ as $n \rightarrow$ $\infty$. Let $\left(x_{0}, y_{0}\right)$ be an arbitrary point below $\mathcal{W}^{s}\left(E_{\bar{x}}\right)$. Then $\left(x_{0}, y_{W}\right) \preceq_{\text {se }}\left(x_{0}, y_{0}\right) \preceq_{\text {se }}\left(x_{0}, 0\right)$ where $y_{W}$ denotes the $y$ coordinate of the point on $\mathcal{W}^{s}\left(E_{\bar{x}}\right)$. Consequently $T^{n}\left(\left(x_{0}, y_{W}\right)\right) \preceq_{\text {se }}$ $T^{n}\left(\left(x_{0}, y_{0}\right)\right) \preceq_{\mathrm{se}} T^{n}\left(\left(x_{0}, 0\right)\right)$, which in view of $T^{n}\left(\left(x_{0}, y_{W}\right)\right) \rightarrow E_{\bar{x}}$ and $T^{n}\left(\left(x_{0}, 0\right)\right) \rightarrow E_{\bar{x}}$ as $n \rightarrow \infty$ implies that $T^{n}\left(\left(x_{0}, y_{0}\right)\right) \rightarrow E_{\bar{x}}$ as $n \rightarrow \infty$.

Let $\left(x_{0}, y_{0}\right)$ be an arbitrary point above $\mathcal{W}^{s}\left(E_{\bar{x}}\right)$. If $x_{0} \geq \bar{x}$, then $\left(0, y_{0}\right) \preceq_{\text {se }}\left(x_{0}, y_{0}\right) \preceq_{\text {se }}$ $\left(x_{0}, y_{W}\right)$ where $y_{W}$ denotes the $y$ coordinate of the point on $\mathcal{W}^{s}\left(E_{\bar{x}}\right)$. Consequently, $T^{n}\left(\left(0, y_{0}\right)\right) \preceq_{\text {se }} T^{n}\left(\left(x_{0}, y_{0}\right)\right) \preceq_{\text {se }} T^{n}\left(\left(x_{0}, y_{W}\right)\right)$, which in view of $T^{n}\left(\left(x_{0}, y_{W}\right)\right) \rightarrow E_{\bar{x}}$ and $T^{n}\left(\left(0, y_{0}\right)\right) \rightarrow E_{0}$ as $n \rightarrow \infty$ implies that $T^{n}\left(\left(x_{0}, y_{0}\right)\right) \rightarrow(x, 0), x<\bar{x}$ as $n \rightarrow \infty$. Thus $T^{n}\left(\left(x_{0}, y_{0}\right)\right) \rightarrow E_{0}$ as $n \rightarrow \infty$. If $x_{0}<\bar{x}$, then $\left(0, y_{0}\right) \preceq_{\mathrm{se}}\left(x_{0}, y_{0}\right) \preceq_{\mathrm{se}}\left(x_{0}, 0\right)$, which implies $T^{n}\left(\left(0, y_{0}\right)\right) \preceq_{\text {se }} T^{n}\left(\left(x_{0}, y_{0}\right)\right) \preceq_{\text {se }} T^{n}\left(\left(x_{0}, 0\right)\right)$, which in view of $T^{n}\left(\left(x_{0}, 0\right)\right) \rightarrow E_{0}$ and $T^{n}\left(\left(0, y_{0}\right)\right) \rightarrow E_{0}$ as $n \rightarrow \infty$ implies that $T^{n}\left(\left(x_{0}, y_{0}\right)\right) \rightarrow E_{0}$ as $n \rightarrow \infty$.

Another proof of this result follows from Theorem 6, which guarantees the existence and uniqueness of $\mathcal{W}^{s}\left(E_{\bar{x}}\right)$ and the invariance of the regions below and above $\mathcal{W}^{s}\left(E_{\bar{x}}\right)$ and Theorem 10, which guarantees that all solutions converge to an equilibrium point.

\section{Theorem 13}

(a) If $a<2, b>2$, then system (6) has three equilibrium points, $E_{0}, E_{\bar{y}_{+}}, E_{\bar{y}_{-}}$, where the first two are locally stable and the third is a saddle point. The basins of attraction of the three equilibrium points are given as

$$
\begin{aligned}
& B\left(E_{0}(0,0)\right)=\left\{\left(x_{0}, y_{0}\right): \text { points below } \mathcal{W}^{s}\left(E_{\bar{y}_{-}}\right)\right\}, \\
& B\left(E_{\bar{y}_{+}}\left(0, \bar{y}_{+}\right)\right)=\left\{\left(x_{0}, y_{0}\right) \text { : points above } \mathcal{W}^{s}\left(E_{\bar{y}_{-}}\right)\right\},
\end{aligned}
$$

where $\mathcal{W}^{s}(E)$ denotes the global stable manifold guaranteed by Theorem 6 .

(b) Similarly, if $a>2, b<2$, the basins of attraction of the equilibrium points are given as

$$
\begin{aligned}
& B\left(E_{0}(0,0)\right)=\left\{\left(x_{0}, y_{0}\right): \text { points above } \mathcal{W}^{s}\left(E_{\bar{x}_{-}}\right)\right\}, \\
& B\left(E_{\bar{x}_{+}}\left(\bar{x}_{+}, 0\right)\right)=\left\{\left(x_{0}, y_{0}\right) \text { : points below } \mathcal{W}^{s}\left(E_{\bar{x}_{-}}\right)\right\} .
\end{aligned}
$$

(c) If $a=b=2$, then system (6) has three equilibrium points, $E_{0}, E_{\bar{x}}, E_{\bar{y}}$, where $E_{0}$ is locally stable and the remaining two are non-hyperbolic of stable type. The basins of 
attraction of three equilibrium points are given as

$$
\begin{aligned}
& B\left(E_{0}(0,0)\right)=\left\{\left(x_{0}, y_{0}\right): \text { region bounded by } \mathcal{W}^{s}\left(E_{\bar{x}}\right) \text { and } \mathcal{W}^{s}\left(E_{\bar{y}}\right)\right\}, \\
& B\left(E_{\bar{y}}\right)=\left\{\left(x_{0}, y_{0}\right) \text { : points above } \mathcal{W}^{s}\left(E_{\bar{y}}\right)\right\}, \\
& B\left(E_{\bar{x}}\right)=\left\{\left(x_{0}, y_{0}\right) \text { : points below } \mathcal{W}^{s}\left(E_{\bar{x}}\right)\right\},
\end{aligned}
$$

where $\mathcal{W}^{s}(E)$ denotes corresponding global stable manifold.

Proof We present the proof in case (a) only. The proof in case (b) is similar.

Local stability of the equilibrium points follows from Theorem 8 .

In view of Theorem 10 all solutions converge to an equilibrium solution. Furthermore, all conditions of Theorem 6 are satisfied, which guarantee the existence of the manifold $\mathcal{W}^{s}\left(E_{\bar{y}_{-}}\right)$, which is the graph of a continuous increasing function and such that both regions, below and above it are invariant. In addition the basin of attraction of $E_{\bar{y}_{-}}$is exactly $\mathcal{W}^{s}\left(E_{\bar{y}_{-}}\right)$. Thus, both regions, below and above $\mathcal{W}^{s}\left(E_{\bar{y}_{-}}\right)$are invariant and contain exactly one equilibrium point and all solutions there are convergent. Consequently the conclusion of the theorem follows.

Let us consider case (c). The existence and the properties of the manifolds $\mathcal{W}^{s}\left(E_{\bar{y}}\right)$ and $\mathcal{W}^{s}\left(E_{\bar{x}}\right)$, as well as the invariance of the regions above $\mathcal{W}^{s}\left(E_{\bar{y}}\right)$, between $\mathcal{W}^{s}\left(E_{\bar{y}}\right)$ and $\mathcal{W}^{s}\left(E_{\bar{x}}\right)$ and below $\mathcal{W}^{s}\left(E_{\bar{x}}\right)$ is guaranteed by Theorem 6 . Since the regions above $\mathcal{W}^{s}\left(E_{\bar{y}}\right)$ and below $\mathcal{W}^{s}\left(E_{\bar{x}}\right)$ contains only one equilibrium point in view of Theorem 10 all solutions that start in those regions converge to $E_{\bar{y}}$ and $E_{\bar{x}}$, respectively.

Now, let $\left(x_{0}, y_{0}\right)$ be an arbitrary point between $\mathcal{W}^{s}\left(E_{\bar{y}}\right)$ and $\mathcal{W}^{s}\left(E_{\bar{x}}\right)$. First assume that $x_{0}<\bar{x}, y_{0}<\bar{y}$. Then $\left(0, y_{0}\right) \preceq_{\text {se }}\left(x_{0}, y_{0}\right) \preceq_{\text {se }}\left(x_{0}, 0\right)$ which implies $T^{n}\left(0, y_{0}\right) \preceq_{\text {se }} T^{n}\left(x_{0}, y_{0}\right) \preceq_{\text {se }}$ $T^{n}\left(x_{0}, 0\right)$. In view of $T^{n}\left(0, y_{0}\right) \rightarrow E_{0}, T^{n}\left(0, x_{0}\right) \rightarrow E_{0}$ as $n \rightarrow \infty$ we conclude that $T^{n}\left(x_{0}, y_{0}\right) \rightarrow E_{0}$ as $n \rightarrow \infty$. Next assume that $x_{0}<\bar{x}, y_{0}<\bar{y}$ is not satisfied. Then there exist points $\left(x_{l}, y_{l}\right) \in \mathcal{W}^{s}\left(E_{\bar{y}}\right),\left(x_{u}, y_{u}\right) \in \mathcal{W}^{s}\left(E_{\bar{x}}\right)$ such that $\left(x_{l}, y_{l}\right) \preceq_{\text {se }}\left(x_{0}, y_{0}\right) \preceq_{\text {se }}\left(x_{u}, y_{u}\right)$ which implies $T^{n}\left(x_{l}, y_{l}\right) \preceq_{\text {se }} T^{n}\left(x_{0}, y_{0}\right) \preceq_{\text {se }} T^{n}\left(x_{u}, y_{u}\right)$. Since $T^{n}\left(x_{l}, y_{l}\right) \rightarrow E_{\bar{y}}, T^{n}\left(x_{u}, y_{u}\right) \rightarrow E_{\bar{x}}$ as $n \rightarrow \infty$ we conclude that $T^{n}\left(x_{0}, y_{0}\right)$ eventually enters the ordered interval $I\left(E_{\bar{y}}, E_{\bar{x}}\right)=$ $\{(x, y): 0 \leq x \leq \bar{x}, 0 \leq y \leq \bar{y}\}$. Since the map $T$ is strongly competitive it will eventually enter the interior of $I\left(E_{\bar{y}}, E_{\bar{x}}\right)$ and then, as we just showed, will converge to $E_{0}$.

See Figure 4 for visual illustration of Theorems 11-13.

\section{Theorem 14}

(a) If $a>2, b=2$, then system (6) has four equilibrium points, $E_{0}, E_{\bar{x}_{+}}, E_{\bar{x}_{-}}, E_{\bar{y}}$, where the first two are locally asymptotically stable, the third is a saddle point and the fourth is non-hyperbolic of the stable type. The basins of attraction of the four equilibrium points are given as

$$
\begin{aligned}
& B\left(E_{0}\right)=\left\{\left(x_{0}, y_{0}\right): \text { region bounded by } \mathcal{W}^{s}\left(E_{\bar{x}_{-}}\right) \text {and } \mathcal{W}^{s}\left(E_{\bar{y}}\right)\right\}, \\
& B\left(E_{\bar{y}}\right)=\left\{\left(x_{0}, y_{0}\right) \text { : points above } \mathcal{W}^{s}\left(E_{\bar{y}}\right)\right\}, \\
& B\left(E_{\bar{x}_{+}}\right)=\left\{\left(x_{0}, y_{0}\right) \text { : points below } \mathcal{W}^{s}\left(E_{\bar{x}_{-}}\right)\right\}, \\
& B\left(E_{\bar{x}_{-}}\right)=\mathcal{W}^{s}\left(E_{\bar{x}_{-}}\right),
\end{aligned}
$$

where $\mathcal{W}^{s}(E)$ denotes the global stable manifold guaranteed by Theorem 6 . 

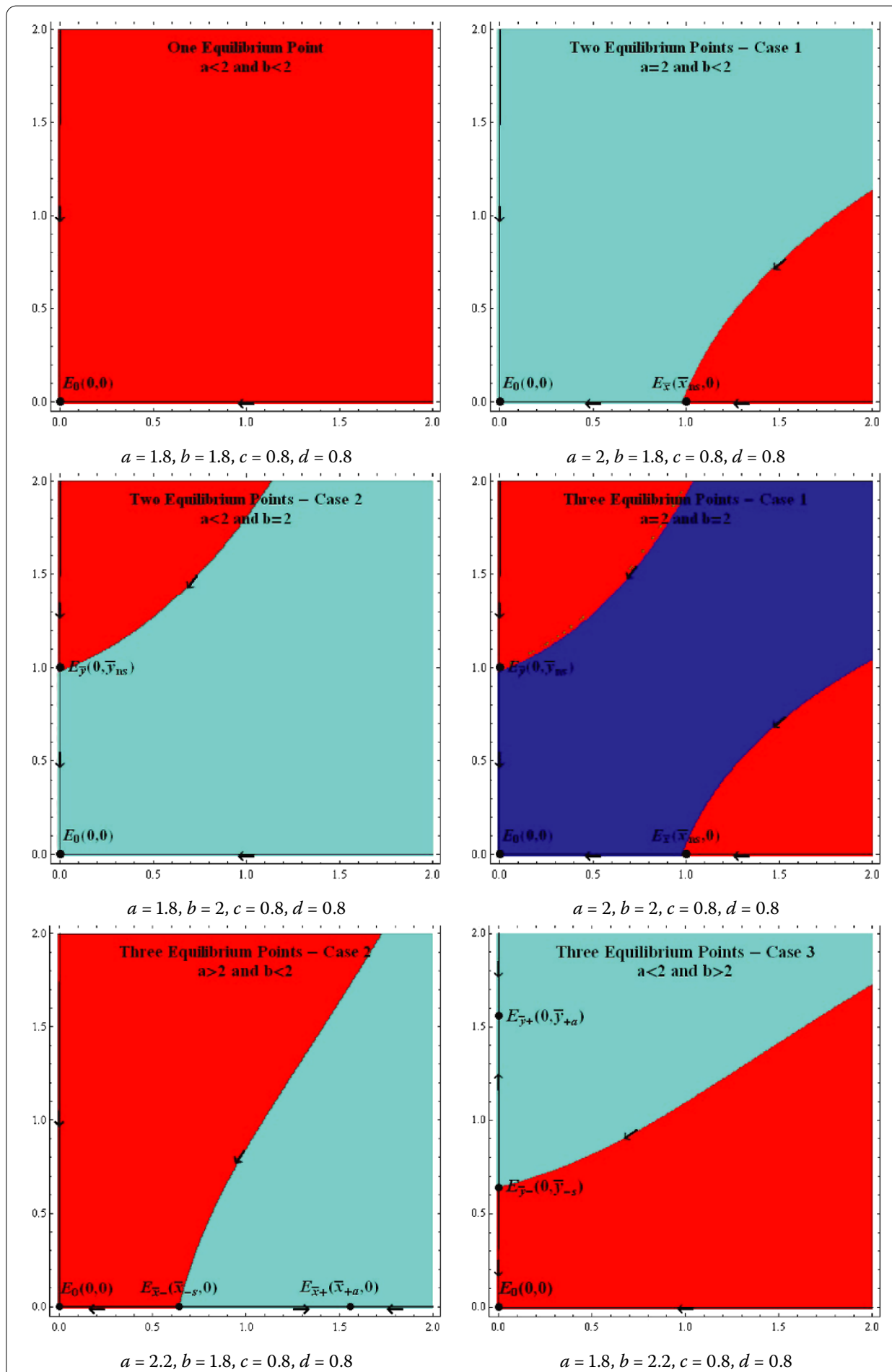

Figure 4 Global stability and basins of attraction of system (6) in cases of one, two, and three equilibrium points. 
(b) Similarly, if $a=2, b>2$, then system (6) has four equilibrium points $E_{0}, E_{\bar{y}_{+}}, E_{\bar{y}_{-}}, E_{\bar{x}}$, where the first two are locally asymptotically stable, the third is a saddle point and the fourth one is non-hyperbolic of the stable type. The basins of attraction are given as

$$
\begin{aligned}
& B\left(E_{0}\right)=\left\{\left(x_{0}, y_{0}\right): \text { region bounded by } \mathcal{W}^{s}\left(E_{\bar{y}_{-}}\right) \text {and } \mathcal{W}^{s}\left(E_{\bar{x}}\right)\right\}, \\
& B\left(E_{\bar{x}}\right)=\left\{\left(x_{0}, y_{0}\right): \text { points below } \mathcal{W}^{s}\left(E_{\bar{x}}\right)\right\}, \\
& B\left(E_{\bar{y}_{+}}\right)=\left\{\left(x_{0}, y_{0}\right): \text { points above } \mathcal{W}^{s}\left(E_{\bar{y}_{-}}\right)\right\}, \\
& B\left(E_{\bar{y}_{-}}\right)=\mathcal{W}^{s}\left(E_{\bar{y}_{-}}\right) .
\end{aligned}
$$

Proof We present the proof in case (a) only. The proof in case (b) is similar. Local stability of the equilibrium points follows from Theorem 8 . The proof for the basin of attraction $B\left(E_{\bar{y}}\right)$ is identical to the proof of the corresponding part of Theorem 12. Let $\left(x_{0}, y_{0}\right)$ be an arbitrary point below $\mathcal{W}^{s}\left(E_{\bar{x}_{-}}\right)$. Then $\left(x_{0}, y_{W}\right) \preceq_{\text {se }}\left(x_{0}, y_{0}\right) \preceq_{\text {se }}\left(x_{0}, 0\right)$ where $y_{W}$ denotes the $y$ coordinate of the point on $\mathcal{W}^{s}\left(E_{\bar{x}_{-}}\right)$. Consequently $T^{n}\left(\left(x_{0}, y_{W}\right)\right) \preceq_{\text {se }} T^{n}\left(\left(x_{0}, y_{0}\right)\right) \preceq_{\text {se }}$ $T^{n}\left(\left(x_{0}, 0\right)\right)$, which in view of $T^{n}\left(\left(x_{0}, y_{W}\right)\right) \rightarrow E_{\bar{x}_{-}}$and $T^{n}\left(\left(x_{0}, 0\right)\right) \rightarrow E_{\bar{x}_{+}}$as $n \rightarrow \infty$ implies that $T^{n}\left(\left(x_{0}, y_{0}\right)\right) \rightarrow E_{\bar{x}_{+}}$as $n \rightarrow \infty$. We also used the fact that the stable manifold $\mathcal{W}^{s}\left(E_{\bar{x}_{-}}\right)$ is unique and represents the basin of attraction of the point $E_{\bar{x}_{-}}$.

Finally, let $\left(x_{0}, y_{0}\right)$ be an arbitrary point between $\mathcal{W}^{s}\left(E_{\bar{y}}\right)$ and $\mathcal{W}^{s}\left(E_{\bar{x}_{-}}\right)$. First assume that $x_{0}<\bar{x}_{-}, y_{0}<\bar{y}$. Then $\left(0, y_{0}\right) \preceq_{\text {se }}\left(x_{0}, y_{0}\right) \preceq_{\text {se }}\left(x_{0}, 0\right)$ which implies $T^{n}\left(0, y_{0}\right) \preceq_{\text {se }} T^{n}\left(x_{0}, y_{0}\right) \preceq_{\text {se }}$ $T^{n}\left(x_{0}, 0\right)$. In view of $T^{n}\left(0, y_{0}\right) \rightarrow E_{0}, T^{n}\left(0, x_{0}\right) \rightarrow E_{0}$ as $n \rightarrow \infty$ we conclude that $T^{n}\left(x_{0}, y_{0}\right) \rightarrow E_{0}$ as $n \rightarrow \infty$. Next assume that $x_{0}<\bar{x}_{-}, y_{0}<\bar{y}$ is not satisfied. Then there exist points $\left(x_{l}, y_{l}\right) \in \mathcal{W}^{s}\left(E_{\bar{y}}\right),\left(x_{u}, y_{u}\right) \in \mathcal{W}^{s}\left(E_{\bar{x}_{-}}\right)$such that $\left(x_{l}, y_{l}\right) \preceq_{\text {se }}\left(x_{0}, y_{0}\right) \preceq_{\text {se }}\left(x_{u}, y_{u}\right)$ which implies $T^{n}\left(x_{l}, y_{l}\right) \preceq_{\mathrm{se}} T^{n}\left(x_{0}, y_{0}\right) \preceq_{\mathrm{se}} T^{n}\left(x_{u}, y_{u}\right)$. Since $T^{n}\left(x_{l}, y_{l}\right) \rightarrow E_{\bar{y}}, T^{n}\left(x_{u}, y_{u}\right) \rightarrow E_{\bar{x}_{-}}$as $n \rightarrow \infty$ we conclude that $T^{n}\left(x_{0}, y_{0}\right)$ eventually enters the ordered interval $I\left(E_{y}, E_{\bar{x}_{-}}\right)=$ $\left\{(x, y): 0 \leq x \leq \bar{x}_{-}, 0 \leq y \leq \bar{y}\right\}$. Since the map $T$ is strongly competitive it will eventually enters the interior of $I\left(E_{\bar{y}}, E_{\bar{x}_{-}}\right)$and then, as we just showed, will converge to $E_{0}$.

Theorem 15 Assume that $a>2, b>2$ and that system (6) has five equilibrium points. Three of these equilibrium points are locally asymptotically stable, $E_{0}, E_{\bar{x}_{+}}, E_{\bar{y}_{+}}$, and two are saddle points, $E_{\bar{x}_{-}}, E_{\bar{y}_{-}}$.

The basins of attraction of the equilibrium points are given as

$$
\begin{aligned}
& B\left(E_{0}\right)=\left\{\left(x_{0}, y_{0}\right) \text { : region bounded by } \mathcal{W}^{s}\left(E_{\bar{x}_{-}}\right) \text {and } \mathcal{W}^{s}\left(E_{\bar{y}_{-}}\right)\right\}, \\
& B\left(E_{\bar{x}_{+}}\right)=\left\{\left(x_{0}, y_{0}\right) \text { : region bounded by } \mathcal{W}^{s}\left(E_{\bar{x}_{-}}\right) \text {and the } x \text {-axis }\right\}, \\
& B\left(E_{\bar{y}_{+}}\right)=\left\{\left(x_{0}, y_{0}\right) \text { : region bounded by } \mathcal{W}^{s}\left(E_{\bar{y}_{-}}\right) \text {and the } y \text {-axis }\right\} .
\end{aligned}
$$

The basins of attraction of the saddle equilibrium points $E$ are the corresponding stable manifolds $\mathcal{W}^{s}(E)$.

Proof Local stability of the equilibrium points follows from Theorem 8 . Furthermore, the existence of the stable manifolds $\mathcal{W}^{s}\left(E_{\bar{x}_{-}}\right)$and $\mathcal{W}^{s}\left(E_{\bar{y}_{-}}\right)$with the mentioned properties follows in the same way as in Theorem 13 . The three regions $B\left(E_{0}\right), B\left(E_{\bar{x}_{+}}\right), B\left(E_{\bar{y}_{+}}\right)$are invariant by Theorem 6 and in view of Theorem 10 every solution converges to an equilibrium point. Since the equilibrium points $E_{0}, E_{\bar{x}_{+}}, E_{\bar{y}_{+}}$are locally asymptotically stable and $E_{\bar{x}_{-}}$, $E_{\bar{y}_{-}}$are saddle points, the result follows. 
Theorem 16 Assume that $a>2, b>2$ and that system (6) has six equilibrium points. Three of these equilibrium points are locally asymptotically stable, $E_{0}, E_{\bar{x}_{+}}, E_{\bar{y}_{+}}$, two are saddle points, $E_{\bar{x}_{-}}, E_{\bar{y}_{-}}$, and one interior point, $E_{n u}$, is non-hyperbolic of the unstable type. There exist two invariant curves $\mathcal{C}_{u}$ and $\mathcal{C}_{l}$ emanating from $E_{n u}$ which are graphs of continuous non-decreasing functions such that $\mathcal{C}_{u}$ is above $\mathcal{C}_{l}$.

The basins of attraction of the equilibrium points are given as

$$
\begin{aligned}
& B\left(E_{0}\right)=\left\{\left(x_{0}, y_{0}\right) \text { : region bounded by } \mathcal{W}^{s}\left(E_{\bar{x}_{-}}\right) \text {and } \mathcal{W}^{s}\left(E_{\bar{y}_{-}}\right)\right\}, \\
& B\left(E_{\bar{x}_{+}}\right)=\left\{\left(x_{0}, y_{0}\right) \text { : region bounded by } \mathcal{W}^{s}\left(E_{\bar{x}_{-}}\right) \cup \mathcal{C}_{l} \text { and the } x \text {-axis }\right\}, \\
& B\left(E_{\bar{y}_{+}}\right)=\left\{\left(x_{0}, y_{0}\right) \text { : region bounded by } \mathcal{W}^{s}\left(E_{\bar{y}_{-}}\right) \cup \mathcal{C}_{u} \text { and the } y \text {-axis }\right\}, \\
& B\left(E_{n u}\right)=\left\{\left(x_{0}, y_{0}\right) \text { : region bounded by } \mathcal{C}_{l} \text { and } \mathcal{C}_{u}\right\} .
\end{aligned}
$$

The basins of attraction of the saddle equilibrium points $B\left(E_{\bar{x}_{-}}\right)$and $B\left(E_{\bar{y}_{-}}\right)$are the corresponding stable manifolds $\mathcal{W}^{s}\left(E_{\bar{x}_{-}}\right)$and $\mathcal{W}^{s}\left(E_{\bar{y}_{-}}\right)$, respectively.

Proof Local stability of the equilibrium points follows from Theorem 8. Furthermore, the existence of the stable manifolds $\mathcal{W}^{s}\left(E_{\bar{x}_{-}}\right)$and $\mathcal{W}^{s}\left(E_{\bar{y}_{-}}\right)$with the mentioned properties follows from Theorem 6 . The region $B\left(E_{0}\right)$ is invariant by Theorem 6 and in view of Theorem 10 every solution which starts in that region converges to $E_{0}$. The existence and the properties of the curves $\mathcal{C}_{l}$ and $\mathcal{C}_{u}$ follow from Corollary 1 . Thus the regions $B\left(E_{\bar{x}_{+}}\right)$ and $B\left(E_{\bar{y}_{+}}\right)$are both invariant and so by Theorem 10 every solution which starts in those regions converges to $E_{\bar{x}_{+}}$and $E_{\bar{y}_{+}}$, respectively, since these equilibrium points are locally asymptotically stable. Finally, the set $B\left(E_{n u}\right)$ is invariant by Theorem 7 and by Theorem 10 every solution which starts in that region converges to $E_{n u}$.

Conjecture 1 Based on our numerical experiments we believe that $\mathcal{C}_{l}=\mathcal{C}_{u}$ in Theorem 16 holds.

Theorem 17 Assume that $a>2, b>2$ and that system (6) has seven equilibrium points. Three of these equilibrium points are locally asymptotically stable, $E_{0}, E_{\bar{x}_{+}}, E_{\bar{y}_{+}}$, three are saddle points, $E_{\bar{x}_{-}}, E_{\bar{y}_{-}}, E_{\mathrm{NW}}$ or $E_{\mathrm{SE}}$, and one is a repeller, $E_{\mathrm{SW}}$.

The basins of attraction of the equilibrium points are given as

$$
\begin{aligned}
& B\left(E_{0}\right)=\left\{\left(x_{0}, y_{0}\right): \text { region bounded by } \mathcal{W}^{s}\left(E_{\bar{x}_{-}}\right) \text {and } \mathcal{W}^{s}\left(E_{\bar{y}_{-}}\right)\right\}, \\
& B\left(E_{\bar{x}_{+}}\right)=\left\{\left(x_{0}, y_{0}\right): \text { region below } \mathcal{W}^{s}\left(E_{\bar{x}_{-}}\right) \cup \mathcal{W}^{s}\left(E_{\mathrm{NW}}\right)\right\}, \\
& B\left(E_{\bar{y}_{+}}\right)=\left\{\left(x_{0}, y_{0}\right): \text { region above } \mathcal{W}^{s}\left(E_{\bar{y}_{-}}\right) \cup \mathcal{W}^{s}\left(E_{\mathrm{NW}}\right)\right\} .
\end{aligned}
$$

The basins of attraction of the saddle equilibrium points E are the corresponding stable manifolds $\mathcal{W}^{s}(E)$.

Proof Local stability of all equilibrium points $E_{0}, E_{\bar{x}_{ \pm}}, E_{\bar{y}_{ \pm}}$follows from Theorem 8.

Three regions $B\left(E_{0}\right), B\left(E_{\bar{x}_{+}}\right), B\left(E_{\bar{y}_{+}}\right)$are invariant by Theorem 6 and in view of Theorem 10 every solution converges to an equilibrium. Since the equilibrium points $E_{0}, E_{\bar{x}_{+}}$, $E_{\bar{y}_{+}}$are locally asymptotically stable and $E_{\bar{x}_{-}}, E_{\bar{y}_{-}}, E_{\mathrm{NW}}$ or $E_{\mathrm{SE}}$ are saddle points, the result follows.

See Figure 5 for visual illustration of Theorems 14-17. 

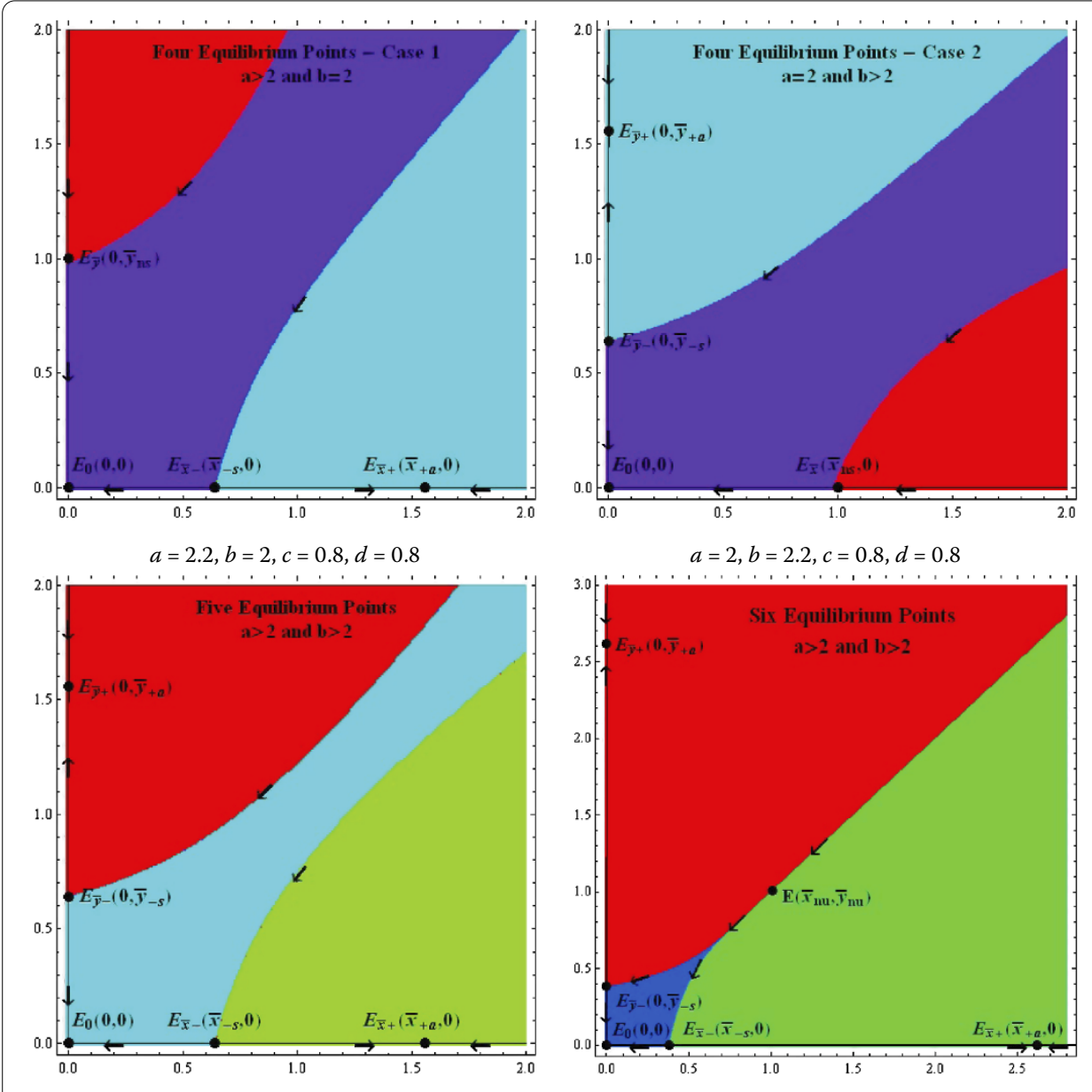

$a=2.2, b=2.2, c=0.8, d=0.8$
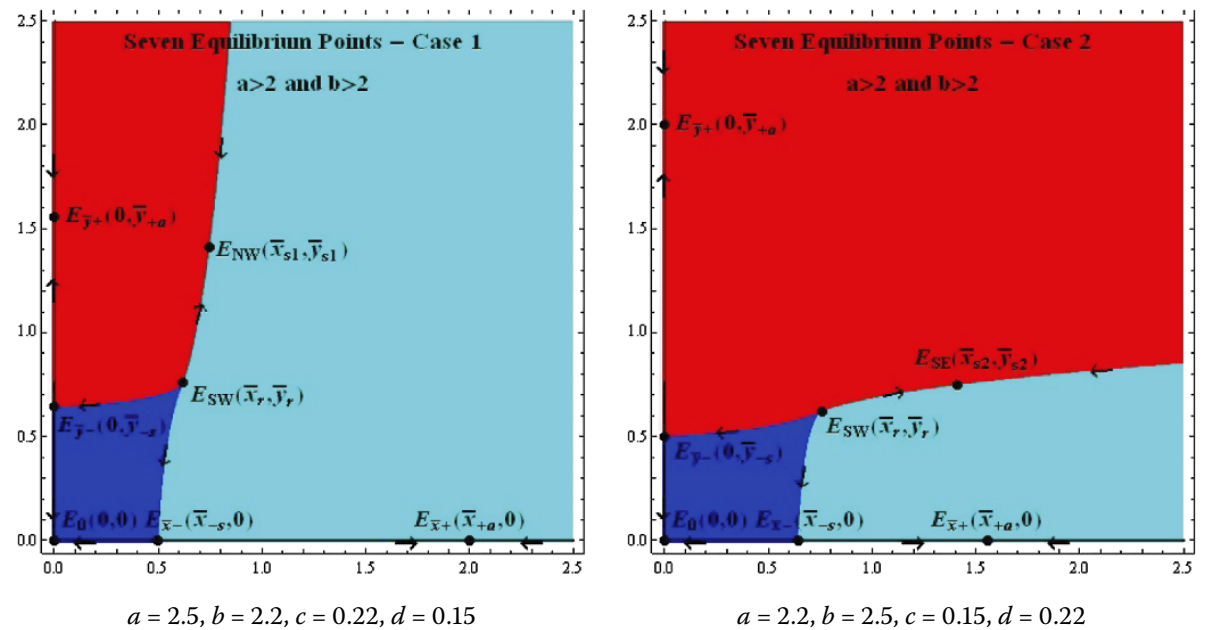

Figure 5 Global stability and basins of attraction of system (6) in cases of four, five, six and seven equilibrium points. 
Theorem 18 Assume that $a>2, b>2$ and that system (6) has eight equilibrium points. Three of these equilibrium points are locally asymptotically stable, $E_{0}, E_{\bar{x}_{+}}, E_{\bar{y}_{+}}$, three are saddle points, $E_{\bar{x}_{-}}, E_{\bar{y}_{-}}, E_{\mathrm{NW}}$ or $E_{\mathrm{SE}}$, one is a repeller, $E_{\mathrm{SW}}$, and one is non-hyperbolic of the stable type $E_{n s}$.

The basins of attraction of the equilibrium points are given as

$$
\begin{aligned}
& B\left(E_{0}\right)=\left\{\left(x_{0}, y_{0}\right) \text { : region bounded by } \mathcal{W}^{s}\left(E_{\bar{x}_{-}}\right) \text {and } \mathcal{W}^{s}\left(E_{\bar{y}_{-}}\right)\right\}, \\
& B\left(E_{\bar{x}_{+}}\right)=\left\{\left(x_{0}, y_{0}\right): \text { region below } \mathcal{W}^{s}\left(E_{\bar{x}_{-}}\right) \cup \mathcal{W}^{s}\left(E_{\mathrm{SE}}\right)\right\}, \\
& B\left(E_{\bar{y}_{+}}\right)=\left\{\left(x_{0}, y_{0}\right) \text { : region above } \mathcal{W}^{s}\left(E_{\bar{y}_{-}}\right) \cup \mathcal{W}^{s}\left(E_{n s}\right)\right\}, \\
& B\left(E_{n s}\right)=\left\{\left(x_{0}, y_{0}\right) \text { : region bounded by } \mathcal{W}^{s}\left(E_{\mathrm{SE}}\right) \text { and } \mathcal{W}^{s}\left(E_{n s}\right)\right\} .
\end{aligned}
$$

The basins of attraction of the saddle equilibrium points E are the corresponding stable manifolds $\mathcal{W}^{s}(E)$.

The same result holds if $E_{\mathrm{SW}}$ is replaced with $E_{\mathrm{NE}}$.

Proof Local stability of the equilibrium points follows from Theorem 8 . The existence and the properties of four manifolds $\mathcal{W}^{s}\left(E_{\bar{x}_{-}}\right), \mathcal{W}^{s}\left(E_{\bar{y}_{-}}\right), \mathcal{W}^{s}\left(E_{\mathrm{SE}}\right), \mathcal{W}^{s}\left(E_{n s}\right)$ follow from Theorem 6.

The four regions $B\left(E_{0}\right), B\left(E_{\bar{x}_{+}}\right), B\left(E_{\bar{y}_{+}}\right), B\left(E_{n s}\right)$ are invariant by Theorem 6 and in view of Theorem 10 every solution converges to an equilibrium point. Since the equilibrium points $E_{0}, E_{\bar{x}_{+}}, E_{\bar{y}_{+}}$are locally asymptotically stable, $E_{n s}$ is non-hyperbolic of stable type and $E_{\bar{x}_{-}}, E_{\bar{y}_{-}}, E_{\mathrm{SE}}$ are saddle points, the result follows.

Theorem 19 Assume that $a>2, b>2$ and that system (6) has nine equilibrium points. Four of these equilibrium points are locally asymptotically stable, $E_{0}, E_{\bar{x}_{+}}, E_{\bar{y}_{+}}, E_{\mathrm{NE}}$, four are saddle points, $E_{\bar{x}_{-}}, E_{\bar{y}_{-}}, E_{\mathrm{NW}}, E_{\mathrm{SE}}$, and one is a repeller, $E_{\mathrm{SW}}$.

The basins of attraction of the equilibrium points are given as

$$
\begin{aligned}
& B\left(E_{0}\right)=\left\{\left(x_{0}, y_{0}\right) \text { : region bounded by } \mathcal{W}^{s}\left(E_{\bar{x}_{-}}\right) \text {and } \mathcal{W}^{s}\left(E_{\bar{y}_{-}}\right)\right\}, \\
& B\left(E_{\bar{x}_{+}}\right)=\left\{\left(x_{0}, y_{0}\right) \text { : region below } \mathcal{W}^{s}\left(E_{\bar{x}_{-}}\right) \cup \mathcal{W}^{s}\left(E_{\mathrm{SE}}\right)\right\}, \\
& B\left(E_{\bar{y}_{+}}\right)=\left\{\left(x_{0}, y_{0}\right) \text { : region above } \mathcal{W}^{s}\left(E_{\bar{y}_{-}}\right) \cup \mathcal{W}^{s}\left(E_{\mathrm{NW}}\right)\right\}, \\
& B\left(E_{\mathrm{NE}}\right)=\left\{\left(x_{0}, y_{0}\right) \text { : region bounded by } \mathcal{W}^{s}\left(E_{\mathrm{SE}}\right) \text { and } \mathcal{W}^{s}\left(E_{\mathrm{NW}}\right)\right\} .
\end{aligned}
$$

The basins of attraction of the saddle equilibrium points E are the corresponding stable manifolds $\mathcal{W}^{s}(E)$.

Proof Local stability of the equilibrium points follows from Theorem 8. The existence and the properties of four manifolds $\mathcal{W}^{s}\left(E_{\bar{x}_{-}}\right), \mathcal{W}^{s}\left(E_{\bar{y}_{-}}\right), \mathcal{W}^{s}\left(E_{\mathrm{SE}}\right), \mathcal{W}^{s}\left(E_{\mathrm{NW}}\right)$ follow from Theorem 6.

The four regions $B\left(E_{0}\right), B\left(E_{\bar{x}_{+}}\right), B\left(E_{\bar{y}_{+}}\right), B\left(E_{\mathrm{NE}}\right)$ are invariant by Theorem 6 and in view of Theorem 10 every solution converges to an equilibrium point. Since the equilibrium points $E_{0}, E_{\bar{x}_{+}}, E_{\bar{y}_{+}}, E_{\mathrm{NE}}$ are locally asymptotically stable and $E_{\bar{x}_{-}}, E_{\bar{y}_{-}}, E_{\mathrm{NW}}$, and $E_{\mathrm{SE}}$ are saddle points, the result follows.

See Figure 6 for visual illustration of Theorems 18, 19. 


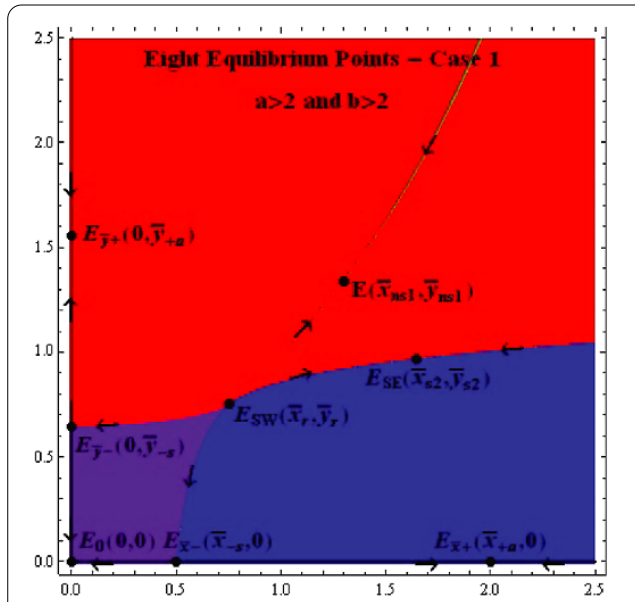

$a=\frac{71,777}{28,730}, b=\frac{22}{10}, c=\frac{92}{221}, d=\frac{318}{3,250}$

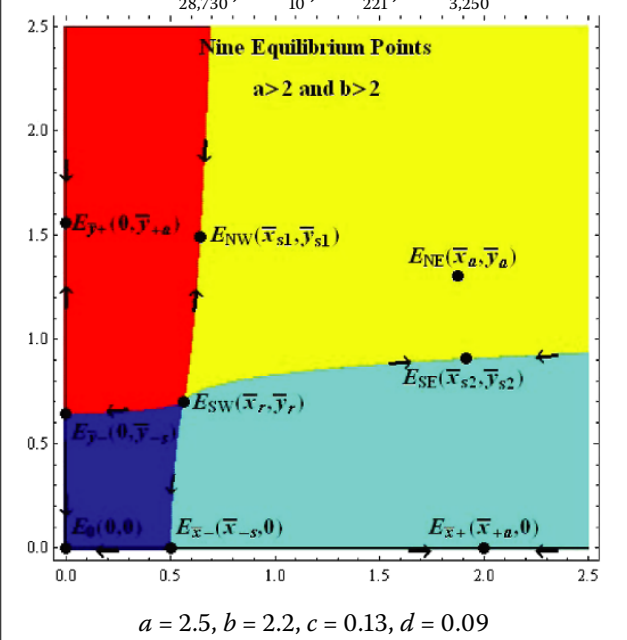

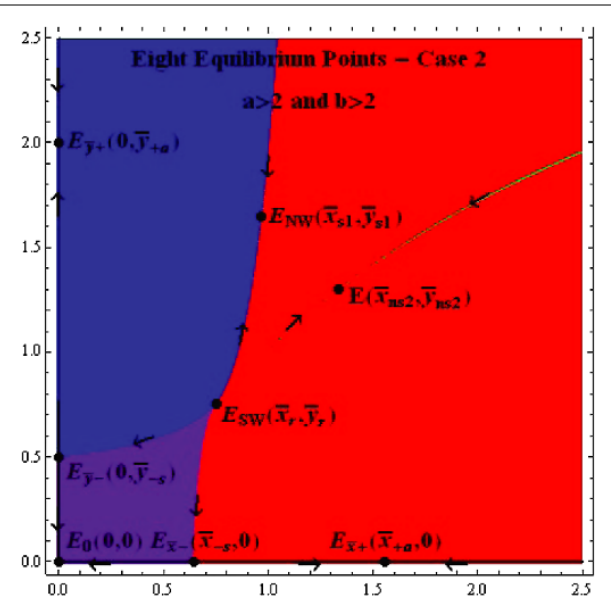

$a=\frac{22}{10}, b=\frac{71,777}{28,730}, c=\frac{318}{3,250}, d=\frac{92}{221}$

Figure 6 Global stability and basins of attraction of system (6) in cases of eight and nine equilibrium points.

\section{Competing interests}

The authors declare that they have no competing interests.

\section{Authors' contributions}

Each of the authors, $A B$ and MRSK, contributed to each part of this work equally and read and approved the final version of the manuscript.

\section{Acknowledgements}

The authors are grateful to two anonymous referees for a number of helpful and constructive suggestions.

\section{Received: 27 August 2014 Accepted: 17 November 2014 Published: 03 Dec 2014}

\section{References}

1. Agarwal, RP: Difference Equations and Inequalities: Theory, Methods, and Applications, 2nd edn. Monographs and Textbooks in Pure and Applied Mathematics, vol. 228. Dekker, New York (2000)

2. Kulenović, MRS, Ladas, G: Dynamics of Second Order Rational Difference Equations. Chapman \& Hall/CRC, Boca Raton (2001)

3. Kulenović, MRS, Merino, O: Discrete Dynamical Systems and Difference Equations with Mathematica. Chapman \& Hall/CRC, Boca Raton (2002)

4. Thieme, HR: Mathematics in Population Biology. Princeton Series in Theoretical and Computational Biology. Princeton University Press, Princeton (2003)

5. Allen, LJS: An Introduction to Mathematical Biology. Prentice Hall, New York (2006)

6. Cushing, JM: The Allee effect in age-structured population dynamics. In: Hallam, TG, Gross, LJ, Levin, SA (eds.) Mathematical Ecology, pp. 479-505. World Scientific, Singapore (1988)

7. Harry, AJ, Kent, CM, Kocic, VL: Global behavior of solutions of a periodically forced sigmoid Beverton-Holt model. J. Biol. Dyn. 6, 212-234 (2012) 
8. Thomson, GG: A proposal for a threshold stock size and maximum fishing mortality rate. In: Smith, SJ, Hunt, JJ, Rivard, D (eds.) Risk Evaluation and Biological Reference Points for Fisheries Management. Canad. Spec. Publ. Fish. Aquat. Sci., vol. 120, pp. 303-320 (1993)

9. Basu, S, Merino, O: On the global behavior of solutions to a planar system of difference equations. Commun. Appl. Nonlinear Anal. 16, 89-101 (2009)

10. Brett, A, Kulenović, MRS: Basins of attraction of equilibrium points of monotone difference equations. Sarajevo J. Math. 5(18)(2), 211-233 (2009)

11. Brett, A, Kulenović, MRS: Basins of attraction for two species competitive model with quadratic terms and the singular Allee effect. Discrete Dyn. Nat. Soc. 2014, Article ID 847360 (2014)

12. Burgić, D, Kalabušić, S, Kulenović, MRS: Nonhyperbolic dynamics for competitive systems in the plane and global period-doubling bifurcations. Adv. Dyn. Syst. Appl. 3, 229-249 (2008)

13. Kulenović, MRS, Merino, O: Invariant manifolds for competitive discrete systems in the plane. Int. J. Bifurc. Chaos 20 , 2471-2486 (2010)

14. Kulenović, MRS, Nurkanović, M: Asymptotic behavior of two dimensional linear fractional system of difference equations. Rad. Mat. 11, 59-78 (2002)

15. Kulenović, MRS, Nurkanović, M: Asymptotic behavior of a competitive system of linear fractional difference equations. Adv. Differ. Equ. 2006, Article ID 19756 (2006)

16. Livadiotis, G, Elaydi, S: General Allee effect in two-species population biology. J. Biol. Dyn. 6, 959-973 (2012)

17. Livadiotis, G, Assas, L, Elaydi, S, Kwessi, E, Ribble, D: Competition models with Allee effects. J. Differ. Equ. Appl. 20, 1127-1151 (2014)

18. Chow, Y, Jang, S: Multiple attractors in a Leslie-Gower competition system with Allee effects. J. Differ. Equ. Appl. 20, 169-187 (2014)

19. Cushing, JM, Levarge, S, Chitnis, N, Henson, SM: Some discrete competition models and the competitive exclusion principle. J. Differ. Equ. Appl. 10, 1139-1152 (2004)

20. Clark, D, Kulenović, MRS, Selgrade, JF: Global asymptotic behavior of a two-dimensional difference equation modelling competition. Nonlinear Anal. TMA 52, 1765-1776 (2003)

21. Franke, JE, Yakubu, A-A: Mutual exclusion versus coexistence for discrete competitive systems. J. Math. Biol. 30, 161-168 (1991)

22. Franke, JE, Yakubu, A-A: Global attractors in competitive systems. Nonlinear Anal. TMA 16, 111-129 (1991)

23. Franke, JE, Yakubu, A-A: Geometry of exclusion principles in discrete systems. J. Math. Anal. Appl. 168, 385-400 (1992)

24. Hassell, MP, Comins, HN: Discrete time models for two-species competition. Theor. Popul. Biol. 9, 202-221 (1976)

25. Hirsch, M, Smith, H: Monotone dynamical systems. In: Handbook of Differential Equations: Ordinary Differential Equations, vol. II, pp. 239-357. Elsevier, Amsterdam (2005)

26. Hirsch, M, Smith, HL: Monotone maps: a review. J. Differ. Equ. Appl. 11, 379-398 (2005)

27. Jiang, H, Rogers, TD: The discrete dynamics of symmetric competition in the plane. J. Math. Biol. 25, 573-596 (1987)

28. Krawcewicz, W, Rogers, TD: Perfect harmony: the discrete dynamics of cooperation. J. Math. Biol. 28, 383-410 (1990)

29. Kulenović, MRS, Merino, O: Competitive-exclusion versus competitive-coexistence for systems in the plane. Discrete Contin. Dyn. Syst., Ser. B 6, 1141-1156 (2006)

30. Kulenović, MRS, Merino, O: Global bifurcation for competitive systems in the plane. Discrete Contin. Dyn. Syst., Ser. B 12, 133-149 (2009)

31. Kulenović, MRS, Nurkanović, M: Global asymptotic behavior of a two dimensional system of difference equations modelling cooperation. J. Differ. Equ. Appl. 9, 149-159 (2003)

32. Kulenović, MRS, Nurkanović, M: Asymptotic behavior of a linear fractional system of difference equations. J. Inequal. Appl. 2005, 127-144 (2005)

33. May, RM: Stability and Complexity in Model Ecosystems. Princeton University Press, Princeton (2001)

34. Smith, HL: Periodic competitive differential equations and the discrete dynamics of competitive maps. J. Differ. Equ. 64, 165-194 (1986)

35. Smith, HL: Planar competitive and cooperative difference equations. J. Differ. Equ. Appl. 3, 335-357 (1998)

36. Yakubu, A-A: The effect of planting and harvesting on endangered species in discrete competitive systems. Math. Biosci. 126, 1-20 (1995)

37. Yakubu, A-A: A discrete competitive system with planting. J. Differ. Equ. Appl. 4, 213-214 (1998)

38. de Mottoni, P, Schiaffino, A: Competition systems with periodic coefficients: a geometric approach. J. Math. Biol. 11, 319-335 (1981)

39. Smith, HL: Periodic solutions of periodic competitive and cooperative systems. SIAM J. Math. Anal. 17, 1289-1318 (1986)

40. Hess, P: Periodic-Parabolic Boundary Value Problems and Positivity. Pitman Research Notes in Mathematics Series, vol. 247. Longman, Harlow (1991)

41. Camouzis, E, Kulenović, MRS, Merino, O, Ladas, G: Rational systems in the plane. J. Differ. Equ. Appl. 15, 303-323 (2009)

42. Kulenović, MRS, Merino, O: Invariant curves of planar competitive and cooperative maps (to appear)

43. Clark, D, Kulenović, MRS: On a coupled system of rational difference equations. Comput. Math. Appl. 43, 849-867 (2002)

44. Yang, L, Hou, X, Zeng, Z: Complete discrimination system for polynomials. Sci. China Ser. E 39(6), 628-646 (1996)

10.1186/1687-1847-2014-307

Cite this article as: Brett and Kulenović: Two species competitive model with the Allee effect. Advances in Difference Equations 2014, 2014:307 